\title{
Principal stress rotation as cause of cyclic mobility
}

Michael Jefferies PEng

Consulting Engineer, Surrey, BC, Canada

Dawn Shuttle PEng, MICE, PhD

Consulting Engineer, Surrey, BC, Canada
Ken Been PEng, MICE, PhD

Golder Associates, Halifax, NS, Canada

Principal stress rotation (PSR) has been known to act as 'loading' on soils since the seminal work of Arthur et al. some 30 years ago, using the directional shear cell. However, the key insight - reflected in the titles of the original papers - that soils yield under constant stress invariants if the principal stresses rotate, has been consistently neglected in virtually all constitutive models of soil as well as in all procedures/methods of geotechnical engineering practice. This neglect is a pity, as a rather simple softening (annealing) of yield surface size, proportional to the rotation of principal stress, captures much of soil behaviour that is measured in the cyclic simple shear test - an idealisation demonstrated in this paper using a minimal extension of the generalised critical state model NorSand (although the idealised effect of PSR could be implemented in any comparable model as the ideas are general). The extended model captures much of the behaviour encountered in the large body of test data on Fraser River sand. Equally, the implementation of PSR annealing makes clear that there is a further loading process for soil: it is suggested that the rotation of the strain increment direction is the missing additional factor. The model is provided as a downloadable, open-source code Excel/ VBA program as a supplement to this paper.

\begin{tabular}{|c|c|c|c|c|c|}
\hline \multirow{2}{*}{\multicolumn{3}{|c|}{$\begin{array}{l}\text { Notation } \\
\text { Subscripts }\end{array}$}} & $\theta$ & \multirow{2}{*}{$\begin{array}{l}{[\mathrm{Rad}]} \\
{\left[\mathrm{FL}^{-2}\right]}\end{array}$} & \multirow{2}{*}{$\begin{array}{l}\text { Lode angle, } \sin (3 \theta)=-13 \cdot 5 \bar{\sigma}_{1} \bar{\sigma}_{2} \bar{\sigma}_{3} / \bar{\sigma}_{q}^{3} \\
\text { principal stresses }\end{array}$} \\
\hline & & & $\sigma_{1,2,3}$ & & \\
\hline c & \multicolumn{2}{|c|}{ critical state } & $\bar{\sigma}_{\mathrm{m}}$ & {$\left[\mathrm{FL}^{-2}\right]$} & mean effective stress $\bar{\sigma}_{\mathrm{m}}=\left(\bar{\sigma}_{1}+\bar{\sigma}_{2}+\bar{\sigma}_{3}\right) / 3$ \\
\hline cy & \multicolumn{2}{|c|}{ cyclic } & $\bar{\sigma}_{q}$ & {$\left[\mathrm{FL}^{-2}\right]$} & deviatoric stress invariant $\bar{\sigma}_{q}=\left(1 / 2\left(\sigma_{1}-\sigma_{2}\right)^{2}+\right.$ \\
\hline & \multicolumn{2}{|c|}{ limit value } & & & $\left.1 / 2\left(\sigma_{2}-\sigma_{3}\right)^{2}+1 / 2\left(\sigma_{3}-\sigma_{1}\right)^{2}\right)^{1 / 2}$ \\
\hline $\begin{array}{l}\mathrm{i} \\
\mathrm{ref}\end{array}$ & \multicolumn{2}{|c|}{$\begin{array}{l}\text { image condition (occurs when } D^{p}=0 \text { for all } \dot{D}^{p} \neq 0 \text { ) } \\
\text { indicates reference stress level; by convention } p_{\text {ref }}=100 \mathrm{kPa}\end{array}$} & $\tau_{\mathrm{xy}}$ & {$\left[\mathrm{FL}^{-2}\right]$} & $\begin{array}{l}\text { horizontal shear stress applied to top of } \\
\text { simple shear apparatus (see Figure 5) }\end{array}$ \\
\hline st & \multicolumn{2}{|c|}{ static } & \multicolumn{3}{|c|}{ Strain variables (dot superscript denotes rate) } \\
\hline te & triaxial compression condition $(\theta=\pi / 6)$ & triaxial extension condition $(\theta=-\pi / 6)$ & $D^{p}$ & {$[-]$} & $\begin{array}{l}\text { plastic dilatancy, as strain rate ratio } \\
\dot{\mathcal{E}}^{p} \dot{\mathcal{E}}_{q}^{p}\end{array}$ \\
\hline $\begin{array}{l}\mathrm{v} \\
\mathrm{x}\end{array}$ & \multicolumn{2}{|c|}{ volume } & $\gamma_{\mathrm{xy}}$ & {$[-]$} & $\begin{array}{l}\text { shear strain reported for simple shear (see } \\
\text { Figure 5) }\end{array}$ \\
\hline y & \multicolumn{2}{|c|}{ vertical } & $\varepsilon_{1,23}$ & [-] & principal strains (assumed coaxial with \\
\hline $\mathrm{Z}$ & \multicolumn{2}{|c|}{ out-of-plane } & & & principal stresses) \\
\hline 0 & \multicolumn{2}{|c|}{ initial condition } & $\dot{\varepsilon}_{q}$ & {$[-]$} & shear strain rate measure work conjugate \\
\hline \multicolumn{3}{|c|}{ Superscripts } & & & with $\bar{\sigma}_{q} \dot{\varepsilon}_{q}=\frac{1}{2}\left[(\sin \theta+\sqrt{3} \cos \theta) \dot{\varepsilon}_{1}\right.$ \\
\hline \multicolumn{3}{|c|}{ Dot "' denotes increment } & & & $-2 \sin \theta \dot{\varepsilon}_{2}+(\sin \theta-\sqrt{3} \cos \theta) \dot{\varepsilon}_{3}$ \\
\hline $\mathrm{p}$ & \multicolumn{2}{|c|}{ plastic } & $\dot{\varepsilon}_{v}$ & {$[-]$} & volumetric strain rate $\dot{\varepsilon}_{v}=\dot{\varepsilon}_{1}+\dot{\varepsilon}_{2}+\dot{\varepsilon}_{3}$ \\
\hline \multicolumn{3}{|c|}{ Stress variables (bar over or' denotes effective) } & \multicolumn{3}{|c|}{ State variables } \\
\hline$p^{\prime}$ & {$\left[\mathrm{FL}^{-2}\right]$} & mean effective stress $\left(=\bar{\sigma}_{\mathrm{m}}\right)$ & e & {$[-]$} & void ratio \\
\hline$q$ & {$\left[\mathrm{FL}^{-2}\right]$} & triaxial deviator stress $q=\sigma_{1}-\sigma_{3}\left(=\bar{\sigma}_{q}\right)$ & $K_{0}$ & {$[-]$} & geostatic stress ratio prior to loading, \\
\hline$u$ & {$\left[\mathrm{FL}^{-2}\right]$} & pore pressure & & & $K_{0}=\bar{\sigma}_{h 0} / \bar{\sigma}_{v 0}$ \\
\hline \multirow[t]{3}{*}{$\alpha$} & \multirow[t]{3}{*}{ [Rad] } & included angle between direction of major & $R$ & {$[-]$} & \multirow{3}{*}{$\begin{array}{l}\text { scalar measure of overconsolidation } \\
\text { state parameter, } \psi=e-e_{\mathrm{c}}\end{array}$} \\
\hline & & principal stress (the ' 1 ' direction) and & $\psi$ & {$[-]$} & \\
\hline & & $y$-coordinate frame of reference & Elas & & \\
\hline \multirow[t]{2}{*}{$\eta$} & & dimensionless shear stress measure as ratio & $E$ & {$\left[\mathrm{FL}^{-2}\right]$} & Young's modulus \\
\hline & & of stress invariants $\eta=\bar{\sigma}_{q} / \bar{\sigma}_{\mathrm{m}}$ & G & {$\left[\mathrm{FL}^{-2}\right]$} & shear modulus \\
\hline
\end{tabular}




$\begin{array}{lll}I_{\mathrm{r}} & {[-]} & \text { soil shear rigidity }\left(=G / \bar{\sigma}_{\mathrm{m}}\right) \\ K & {\left[\mathrm{FL}^{-2}\right]} & \text { bulk modulus } \\ V & {[-]} & \text { Poisson's ratio }\end{array}$

Critical state

\begin{tabular}{|c|c|c|}
\hline$a$ & {$[-]$} & parameter for 'curved CSL' analogous to $\Gamma$ \\
\hline$b$ & {$[-]$} & parameter for 'curved CSL' analogous to $\lambda$ \\
\hline c & {$[-]$} & $\begin{array}{l}\text { parameter for 'curved CSL', an exponent on } \\
\text { stress level }\end{array}$ \\
\hline$M$ & {$[-]$} & $\begin{array}{l}\text { critical friction ratio, } \eta_{\mathrm{c}}=M \text { at the critical } \\
\text { state. Varies with Lode angle, value at } \\
\text { triaxial compression }\left(M_{\mathrm{tc}}\right) \text { taken as reference }\end{array}$ \\
\hline$\Gamma$ & {$[-]$} & $\begin{array}{l}\text { reference void ratio on CSL, conventionally } \\
\text { defined at } p^{\prime}=1 \mathrm{kPa}\end{array}$ \\
\hline$\lambda_{\mathrm{e}}, \lambda_{10}$ & {$[-]$} & $\begin{array}{l}\text { slope of CSL, for semi-log idealisation. } \\
\text { Subscript denotes base of logarithm }\left(\lambda_{10} \sim\right. \\
\left.2 \cdot 3 \lambda_{\mathrm{e}}\right)\end{array}$ \\
\hline
\end{tabular}

NorSand model parameters (in addition to those defining the critical state)

$H \quad$ [-] plastic hardening modulus, may vary with state parameter

$N \quad$ [-] $\quad$ volumetric coupling parameter (from Nova's flow rule)

Z [ $\quad[-] \quad$ plastic softening modulus under principal stress rotation

$\chi \quad[-] \quad$ state-dilatancy parameter, a soil property

\section{Introduction}

Principal stress rotation (PSR) is pervasive in engineering works involving soil, arising in the simplest static loading of a spread footing through to very complicated cyclic rotations during wave loading of offshore structures. PSR is also a central aspect of earthquake loading of soil, with all the consequent issues around the potential for earthquake-induced liquefaction.

The situation in an earthquake is illustrated on Figure 1, focusing on motion in a single vertical plane for simplicity and showing soil overlying bedrock. The earthquake disturbance propagates far faster through rock than soil, so that the soil essentially experiences a vertically propagating motion from the underlying bedrock. It is usual to idealise the soil response as entirely caused by the shear waves so that the 'action' of the earthquake on the soil is taken as cyclically varying horizontal shear stress (often denoted as ' $\tau_{\text {cyc }}$ ') with constant vertical total stress - the widely used site response program SHAKE is based on this analogy. The action of the earthquake is then described as a time-varying ratio of cyclic stress ratio, $\operatorname{CSR}=\tau_{\mathrm{xy}} / \sigma_{\mathrm{y}}$, adopting the reference frame of $\mathrm{x}=$ horizontal and $\mathrm{y}=$ vertical.

Considering the stress state on the element sketched in Figure 1, and continuing with the idealisation of plane strain (both for simplicity and because the test data for validation is plane strain), the stress state on the element of $\left[\sigma_{\mathrm{x}}, \sigma_{\mathrm{y}}, \sigma_{\mathrm{z}}, \tau_{\mathrm{xy}}\right]$ can also be expressed in terms of the principal stresses $\left[\sigma_{1}, \sigma_{2}, \sigma_{3}, \alpha\right]$ where $\alpha$ is the direction between vertical (the y direction) and the direction of the major principal stress $\sigma_{1}$ with $\sigma_{2}=\sigma_{\mathrm{z}}$ (from the plane strain condition). The idealised earthquake loading of Figure 1 involves cyclic variation of $\alpha$ in all situations.

Surprisingly, the parameter $\alpha$ has long been neglected as a contributing factor to soil behaviour, both in terms of understanding the results of laboratory tests and within constitutive models of soil behaviour. Current

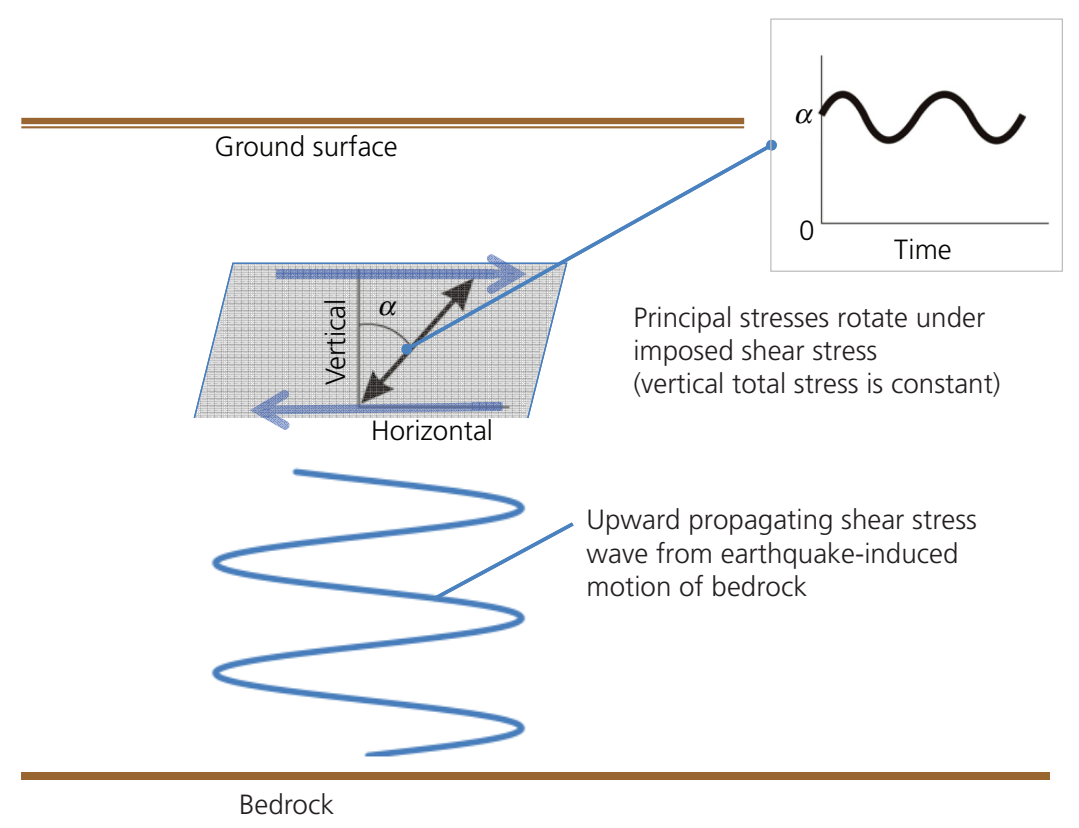

Figure 1. Idealised ground loading by earthquake-induced motion

of bedrock 
understanding of soil behaviour is largely based on the concept of mobilised stress ratio, with the ratio $\eta=\bar{\sigma}_{q} / \bar{\sigma}_{\mathrm{m}}$ (or, equivalently, the mobilised friction angle $\phi_{\mathrm{m}}$ ) being widely used. Simplifying loading paths slightly, increasing $\eta$ implies loading (and thus plastic strains) while decreasing $\eta$ is unloading (and thus elastic strains, although yield in unloading can develop in some circumstances - see Jefferies (1997) for example). Although a change in the principal stress magnitudes usually accompanies PSR, this is not necessarily the case and it is possible to have purely cyclic variations in principal stress direction with constant principal stress magnitudes, and thus constant $\eta$ as well as other stress invariants. Such a loading was investigated by Arthur and co-workers (Arthur et al., 1979, 1980; Wong and Arthur, 1986) in the research group at University College London who developed a directional shear cell (DSC), illustrated in Figure 2(a), in which all principal stresses are measured and their direction can be controlled - in particular, smooth sinusoidal variation of $\alpha$ at near constant stress ratio $\eta$ was imposed (Figure 2(b)). This near-constant $\eta$ loading of dense Leighton Buzzard sand caused strains to accumulate readily with each loading cycle, and the greater the PSR, the greater the plastic straining induced (Figure 2(c)). This testing was then extended to both loose and dense Leighton Buzzard sand with a strong effect of PSR on volumetric strain being found (Figure 3). It is apparent from even casual inspection that PSR has dominated the sand's behaviour and that even rather small stress rotation can suppress dilation. In their conclusion, Wong and Arthur (1986: p. 225) state, 'Cyclic rotation of principal stress directions in sand which causes strain radically alters the behaviour of the material from that seen in shear under constant directions of principal stress'. This is by no means an overstatement of the situation.

The results of Arthur and co-workers have been neglected, anecdotally because of concerns over the validity of their data stemming from the low confining stress limits of the DSC equipment. This neglect is unwarranted as the results of Arthur and co-workers were confirmed just a few years later by a second group in Japan using very different equipment, the hollow cylinder test. Ishihara and Towhata (1983) showed, in tests on loose Toyoura sand, that pure cyclic rotation of constant principal stresses in drained tests at nearly $300 \mathrm{kPa}$ confining stress caused irrecoverable volumetric strain. Yoshimine et al. (1998) provide yet more data using the hollow cylinder test.

There can be no doubt that PSR is fundamental from this experimental data, since any constitutive model based solely on stress invariants will predict no strains in the experiments of Arthur et al. (1980), Ishihara and Towhata (1983), or Yoshimine et al. (1998) - contrary to what is observed. What is needed is to recognise that 'loading' of soil needs to be expressed as the combination of the two measures $\eta$ and $\alpha$ - the seminal insight of Arthur et al. (1979) implied in the title of their paper.

The use of $\{\eta, \alpha\}$ as the loading 'measure' of the soil has been explored in a few constitutive models. Japanese workers pursued models in parallel to their hollow cylinder investigations; Gutierrez et al. (1993) appears to be the most developed example of these workers. That model invoked a stress-dilatancy relation that incorporates non-coaxiality of principal stress and principal plastic strain increment direction, a feature that is perhaps its greatest contribution. However, the effect of changing $\alpha$ was implicit in the loading measure causing yielding and the hardening model was a complicated multi-surface approximation (there was no concept of a critical state dependence of the hardening). Reasonable fits were achieved to the results of hollow cylinder tests, but it is unclear that the model would be satisfactory for drained triaxial compression of loose and dense sands - such a validation was not presented.

A second approach to incorporating $\{\eta, \alpha\}$ into a constitutive model was that of Been et al. (1993), who included PSR within a critical state model. The Been et al. idealisation is shown in Figure 4 and amounts to a Cam-Clay-like yield surface overlain on a rotating frame of reference - the loading vector of $\{\eta, \alpha\}$ is explicit. A key aspect is that the idealisation reduces to the validated model for fixed principal stress directions, but this came with continuing coaxiality of stress and strain increment under PSR. Been et al. presented reasonable fits to the behaviour of Nevada sand in undrained cyclic simple shear (CSS).

This paper is an extension of the Been et al. model. A physical background as to why $\{\eta, \alpha\}$ should be treated as the loading vector is provided, argued from the viewpoint of micromechanics not engineering plasticity, followed by details of how this loading vector is implemented in a general critical state model (NorSand).

Treating $\{\eta, \alpha\}$ as the loading vector necessarily invokes a second plastic hardening modulus for PSR, and thus requires a laboratory test with PSR for calibration - the CSS test is used. In the past decade, this CSS test has gone from only being encountered in research universities to becoming the 'test du jour' for much engineering practice.

Despite the utility of the CSS test for calibrating the effects of PSR, the CSS test is useless for the determination of soil properties: neither $\sigma_{\mathrm{x}}$ nor $\sigma_{\mathrm{z}}$ are measured, so neglecting half the stress tensor (it is not possible to construct a Mohr's circle of conditions in a standard simple shear test). A set of CSS tests necessarily requires complementary triaxial compression testing to determine the soil properties; quantifying how PSR affects soil behaviour requires the soil properties to be previously known. Data sets with both comprehensive triaxial and CSS tests are not common. Here, data on Fraser River sand (FRS) are used, a soil from the lower Fraser Valley area adjacent to Vancouver, Canada. A 'standard' example of this sand has been extensively tested in both CSS and triaxial compression, providing an interesting data set to evaluate a critical state approach to PSR.

The paper presents the basis and effect of PSR within the context of the NorSand model and provides full details on PSR aspects that were only alluded to when they were first introduced (Been et al., 1993). The NorSand/PSR model is simply integrated for CSS with the Euler method, and this is conveniently done with Excel - a spreadsheet (Nor_CSS_R1_r0.xls) is a downloadable adjunct to this paper and provided on the journal web site under the 'Supplements' tab. Commented numerical routines are written 


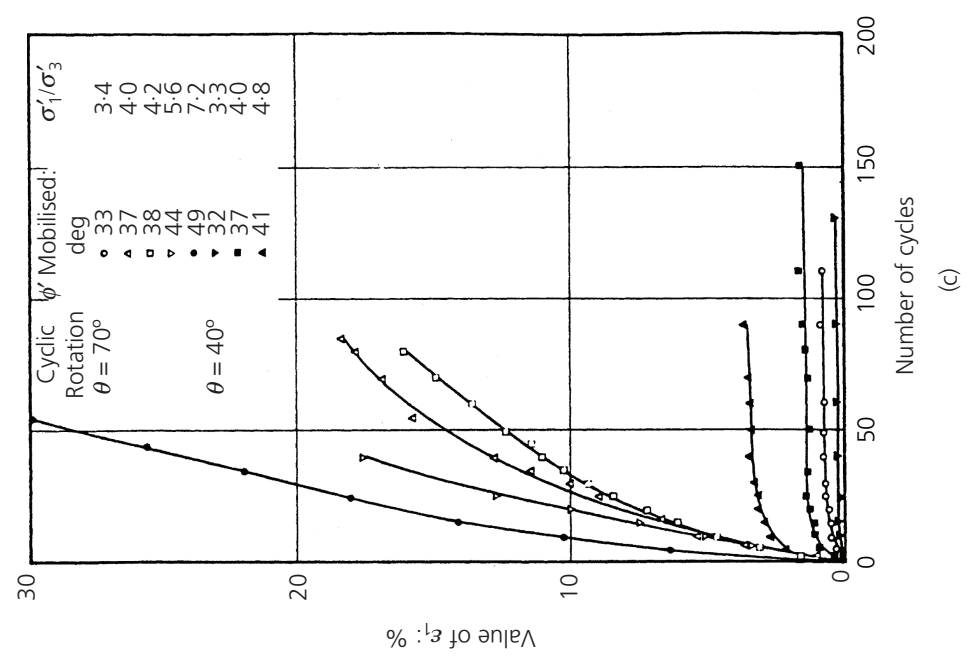

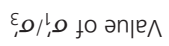
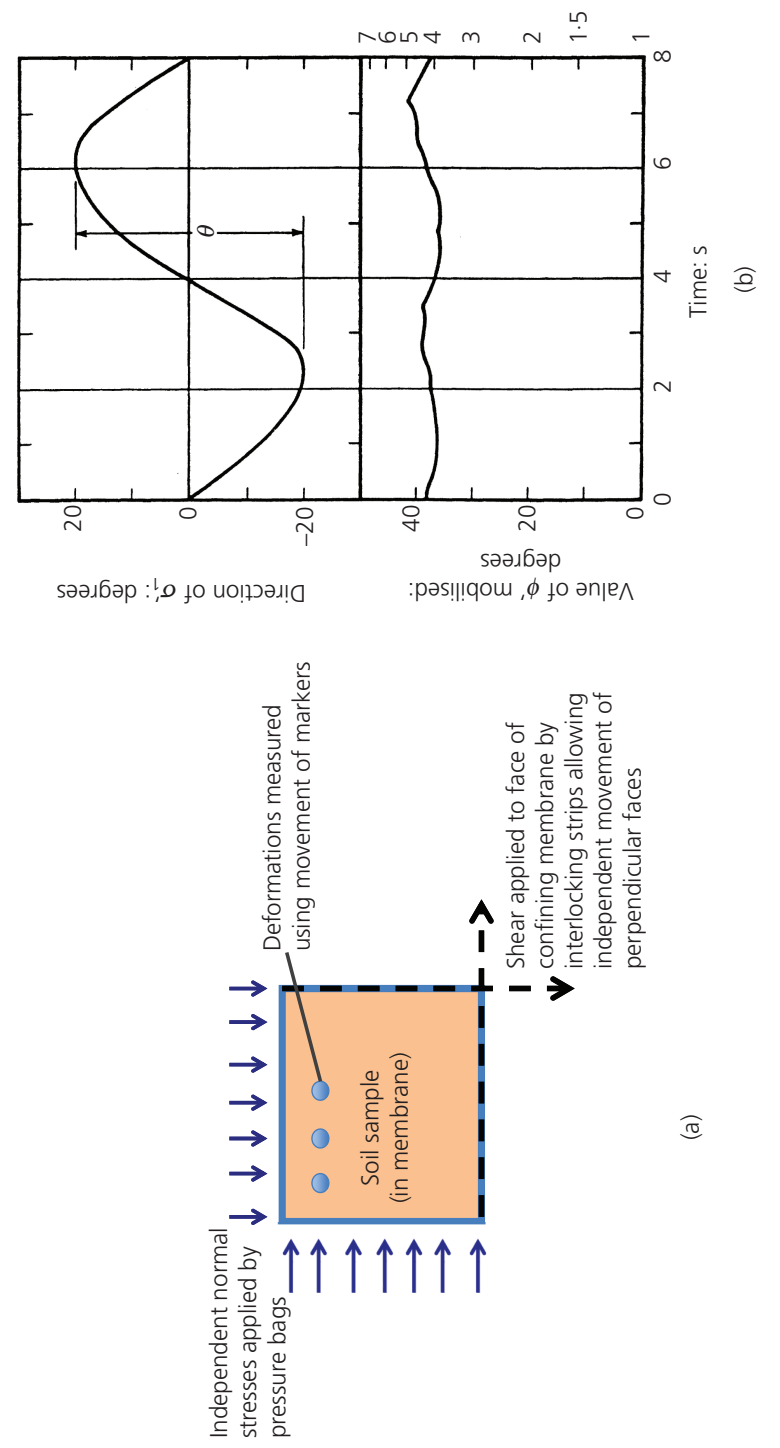


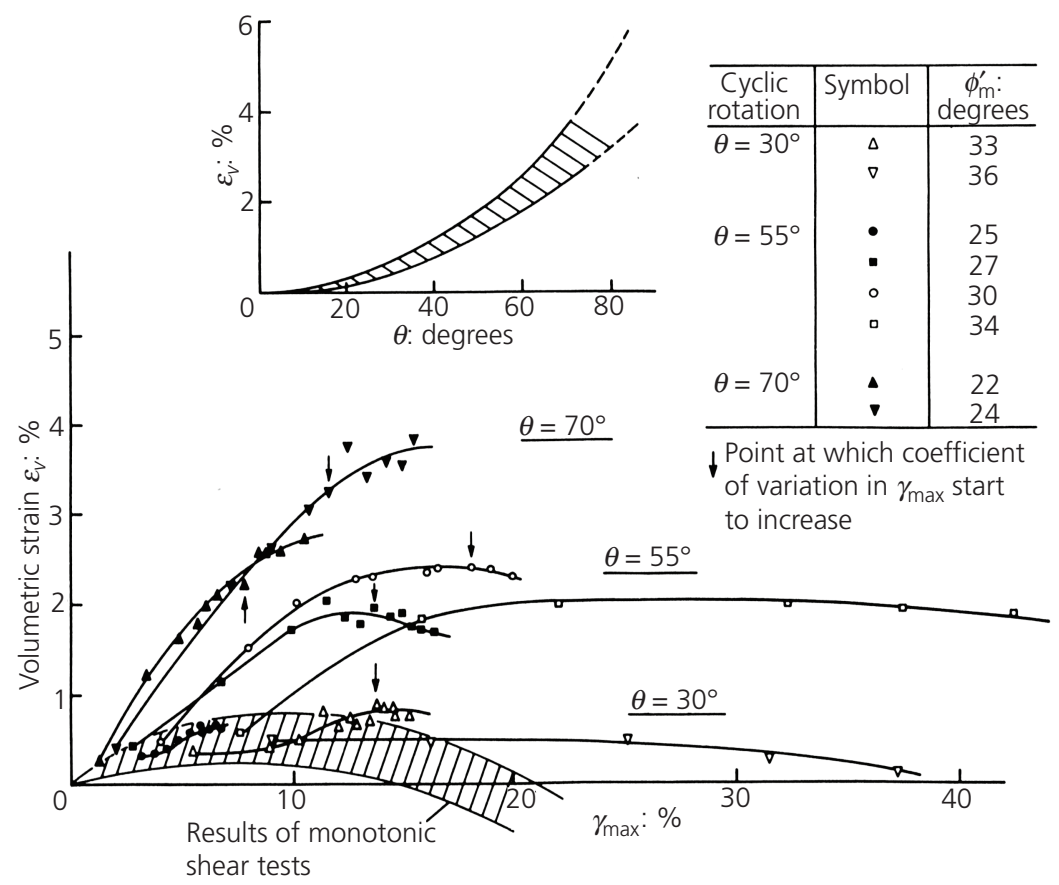

Figure 3. Volumetric strain induced by PSR in dilatant Leighton Buzzard sand (Wong and Arthur, 1986)

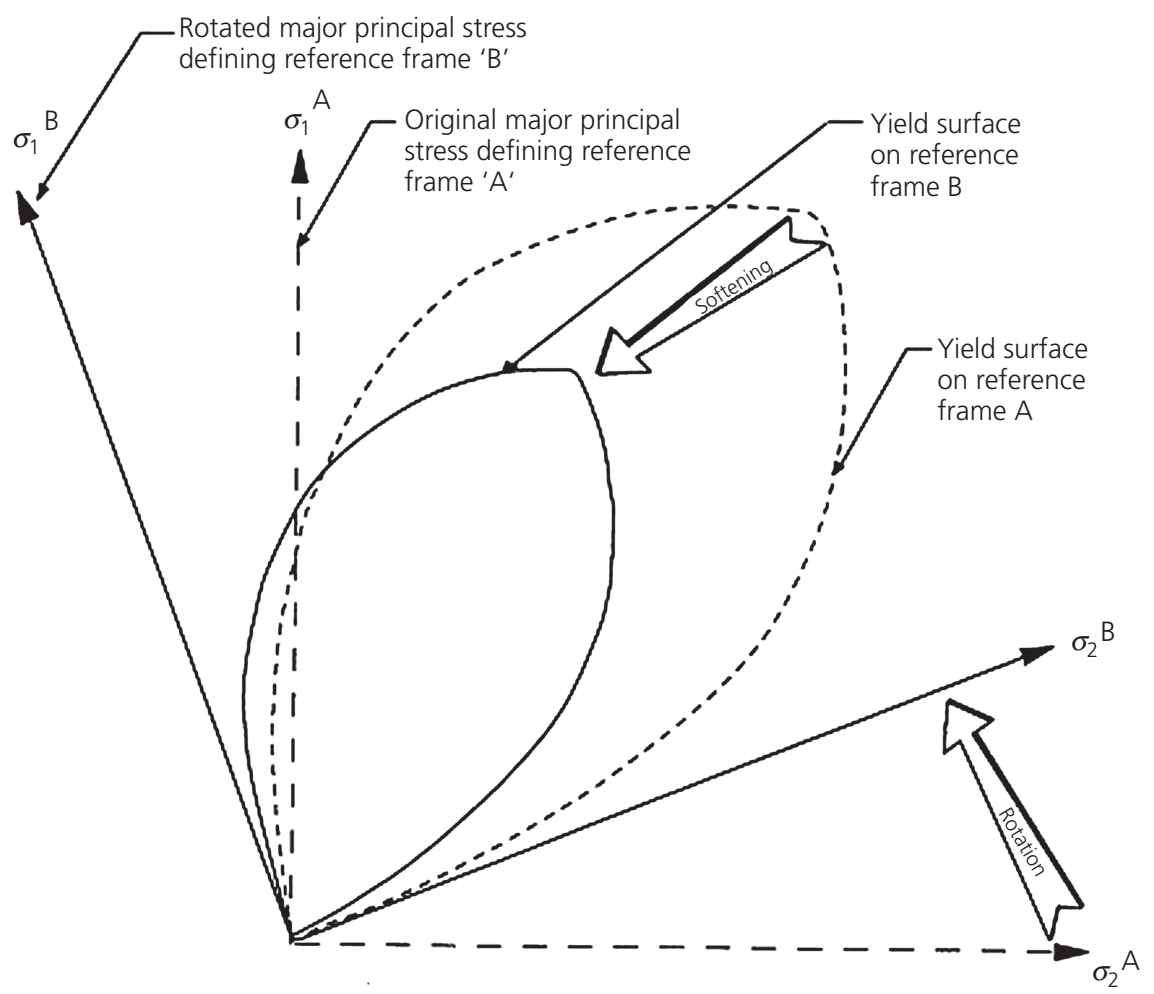

Figure 4. Schematic of idealised yield surface softening induced by PSR (Been et al., 1993) 
in VBA, using near 'plain English' for variable names, and may be found as open-source code under the Excel Visual Basic editor (Menu: /Tools/Macro/Visual Basic Editor) - the subroutine NorCSSu() will be of particular interest for details on how PSR softening was implemented. The spreadsheet requires accepting the 'Enable Macros?' dialog on opening, as Excel treats VBA code as a Macro. The spreadsheet also includes the FRS CSS data and is set up so that the various simulations shown in this paper can be re-run to explore the effects of changing soil properties and so forth (the parameter sets used are embedded in the Excel file).

\section{The CSS test}

The modern version of the simple shear test traces back to the equipment developed by Bjerrum and Landva (1966) that was based on Kjellman's (1951) device. It is sometimes called the NGI or Geonor direct simple shear test and comprises a cylindrical sample (commonly $70 \mathrm{~mm}$ in diameter by about $20 \mathrm{~mm}$ in height) where plane strain is enforced by a reinforced rubber membrane. The sample is loaded by parallel displacement of the confining platens, producing a pure shear loading. 'Undrained' loading in simple shear generally tests dry sand under constant volume conditions (achieved by constraining vertical displacement of the platens) with the changing vertical stress needed to achieve this being taken as equivalent to the excess pore pressure developed during true undrained shear of the sample; Dyvik et al. (1987) report a study supporting this approach. The CSS test takes a standard direct simple shear test and varies the imposed shear load or displacement in a chosen way (often a sinusoidal variation). Figure 5 shows the idealised view of the stress and displacement conditions within the soil during simple shear loading.

Concerns arose over the years about the uniformity of stress and strain conditions within the NGI type of simple shear test. A particular issue is the handling of the complementary shear stress on the lateral confining membrane. Airey and Wood (1987) discuss the issues around direct shear testing as well as an evaluation of the accuracy of average stress and strain as measures of the true soil loading - their work can be summed up as follows: the standard NGI direct shear test has not much worse error in stress and strain than arises with platen effects and sample area correction in the triaxial test. This view is reflected in ASTM Standard D6528 (ASTM, 2007); the reported shear stress is shear load divided by sample area, while shear strain is the shear displacement of the platens divided by the initial sample height. This standard is followed, treating the CSS test results as equivalent to the response of the soil element sketched in Figure 1.

Undrained CSS has two conventions defining the imposed loading. First, a drained shear displacement may be imposed before the start of undrained load cycling. This displacement is referred to as 'static bias' and is usually specified (or quoted) in terms of the resulting static stress ratio, $\mathrm{SSR}=\tau_{\mathrm{st}} / \bar{\sigma}_{v 0}$. Second, the cyclic stress is also quoted or specified in terms of the cyclic stress ratio, $\mathrm{CSR}=\tau_{\mathrm{cyc}} / \bar{\sigma}_{v 0}$. Figure 6(a) illustrates the stress-time series for a test with static bias and with cyclic shear applied at constant rate between the reversal stresses. The CSS test is limited to PSR between $45^{\circ}$ either side of vertical; the effect of static bias is to reduce the range for imposed rotation of principal stress, illustrated in Figure 6(b).

As noted earlier, neither $\sigma_{\mathrm{x}}$ nor $\sigma_{\mathrm{z}}$ are measured. An assumption is therefore needed when formally modelling CSS data. The initial condition $\sigma_{\mathrm{x} 0}=\sigma_{\mathrm{z} 0}$ is assumed and captured as the familiar geostatic stress ratio $K_{0}=\bar{\sigma}_{\mathrm{x} 0} / \bar{\sigma}_{\mathrm{y} 0} ; K_{0}$ is treated as a free variable to best fit the soil model to test data, and with the evolution of $\sigma_{\mathrm{x}}$ and $\sigma_{\mathrm{z}}$ computed (although there is no test data to compare to). Some simulations are shown later to illustrate the sensitivity of the computed results to the assumed $K_{0}$, and thus the precision with which $K_{0}$ can be inferred from other aspects of the soil behaviour in simple shear.

\section{Why PSR is important}

Soil carries load by way of the particle contacts. As load increases and particles move, so does the pattern of contacts that provides the load paths for the forces on the soil - what some refer to as induced anisotropy (e.g. Arthur and Menzies, 1972).

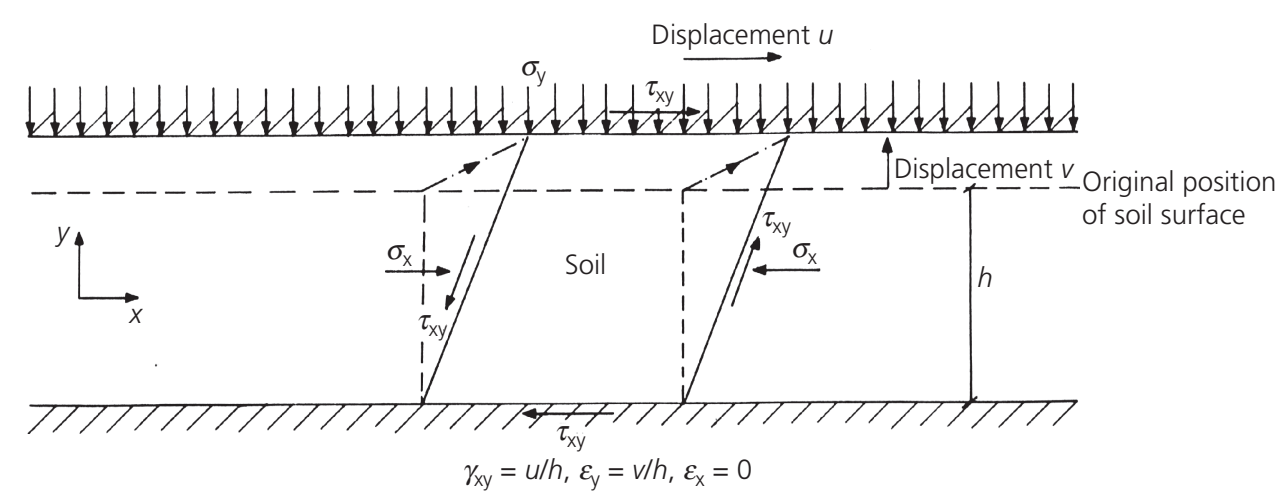

Figure 5. General stress and strain conditions in simple shear (Potts et al., 1987); in the case of undrained loading, the vertical displacement $v=0$ 


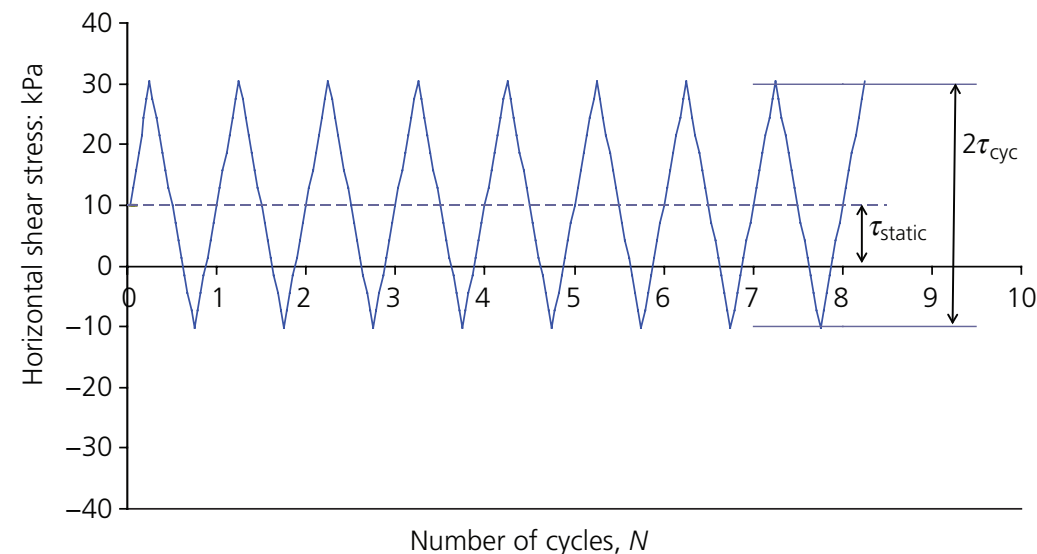

(a)

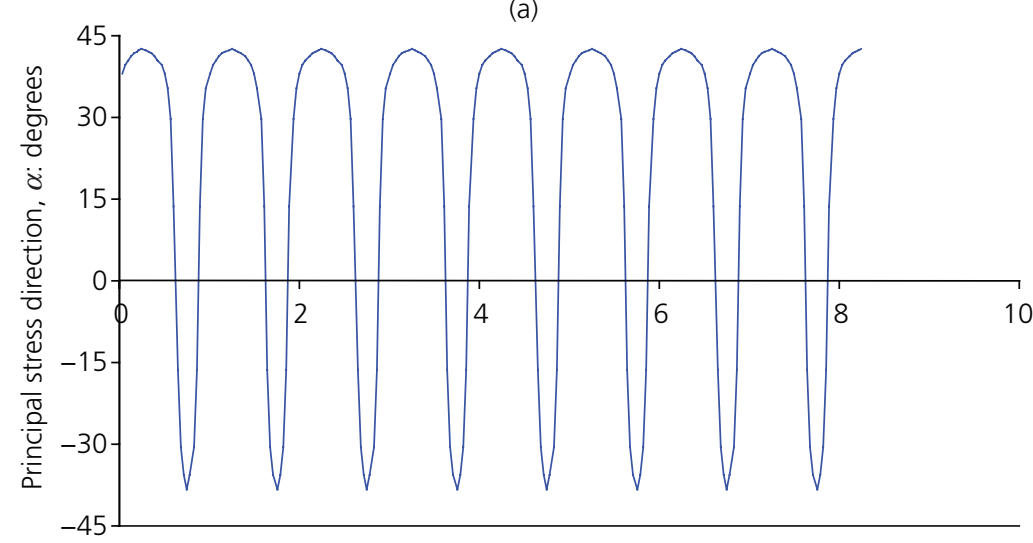

Number of cycles, $N$

(b)

Figure 6. (a) Convention for 'static bias' and 'cyclic stress' in CSS

tests on elastic solid with (b) example of the effect on imposed

principal stress direction

Early investigations of induced anisotropy used photo-elastic discs as an analogue for soil. A notable investigation using this technique was that by De Josselin de Jong and Verruijt (1969), who investigated biaxial compression of the discs; despite being limited to a visual assessment of the load paths, there was clear evidence of a preference for contacts aligned in the direction of the major load (in this case, vertical) to that of the minor load (which was horizontal) perhaps the simplest experimental illustration of induced anisotropy.

More recent investigations of induced anisotropy adopt the distinct-element method, which has become a standard numerical technique for research on the micromechanics of granular materials. Rothenburg and Bathurst (1992) presented the results of numerical simulations of planar assemblies of elliptical particles, assemblies that had qualitative features similar to real sand behaviour. Figure 7(a) shows examples of the arrangement of the elliptical particles, while Figure 7(b) illustrates the computed evolution of the distribution of particle contacts with imposed shear strain. Just as what was found qualitatively with photo-elastic discs, the distinct element approach quantifies how shear strain causes more contacts to develop in the direction of the major principal stress and at the expense of decreasing contacts in the direction of the minor principal stress.

Looking at Figure 7, it is obvious that further vertical loading will have a relatively stiff response. But, if the load is horizontal, or if loaded in an intermediate direction, a completely different, and softer, stress-strain response will arise as the particle arrangement re-adjusts to establish contacts to carry the forces in the direction of loading. PSR matters at a very basic level. So far, so standard - and this view of induced anisotropy might be regarded as widely accepted.

Plasticity theory uses a yield surface to represent the capacity of the assembly of soil particles to carry load. Loads that are within the competence of the assembly with only elastic compression of the soil particles lie within the yield surface. Load combinations that cause the assembly to adjust and form new contacts are on the yield surface, and with the yield surface evolving (hardening) to reflect the formation of new contacts. Yield surfaces must have a notion of their 'size' in stress space. Restricting attention to yield surfaces that have a constant shape allows yield surface size to be described by a single stress measure; 


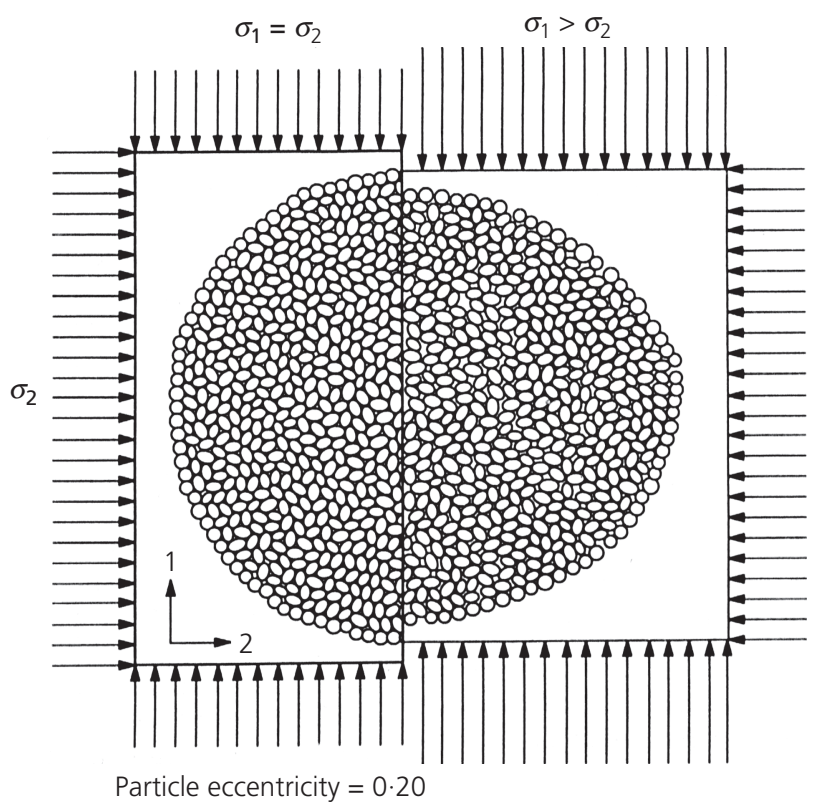

(a)

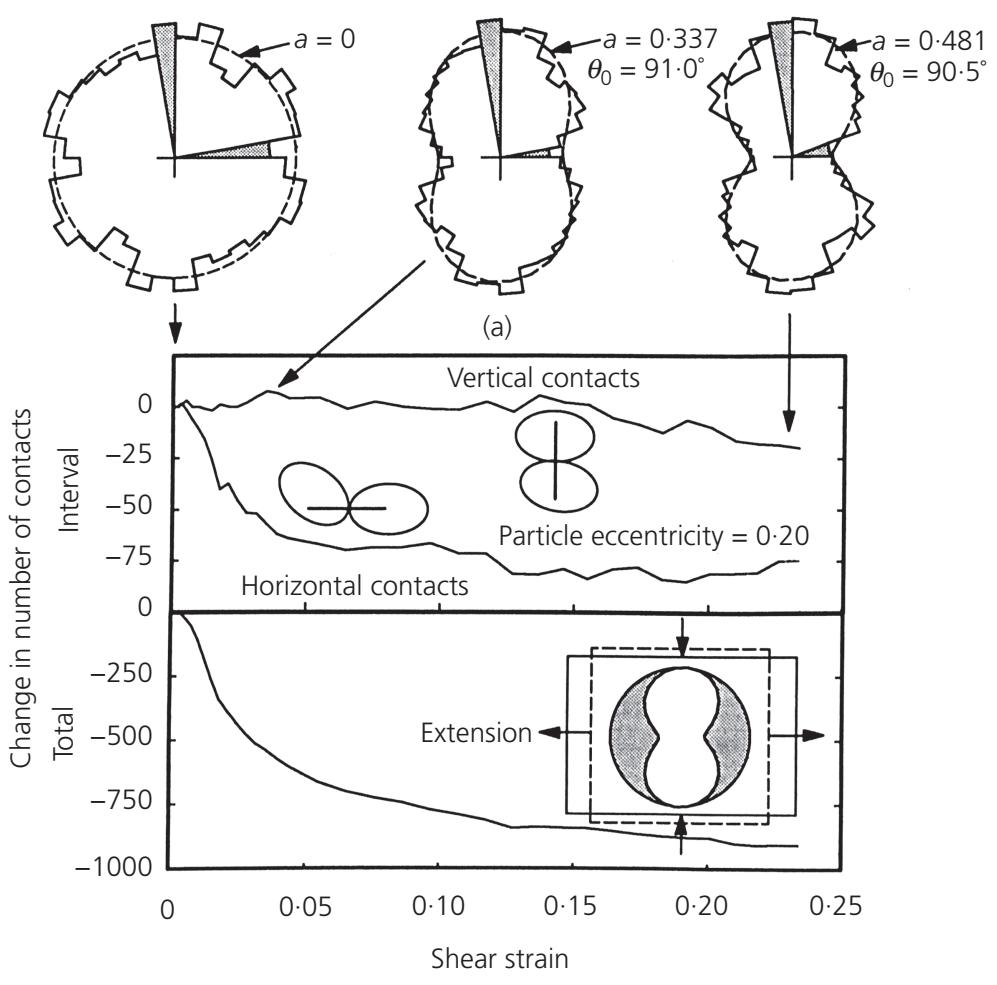

(b)

Figure 7. Biaxial compression of elliptical discs showing evolution of particle contact directions (after Rothenburg and Bathurst, 1992); (a) arrangement of particles before and after shearing by biaxial compression; (b) evolution of distribution of particle contact orientations during shear 
in the case of critical state models this measure is the mean effective stress $\sigma_{\text {mi }}^{\prime}$ for the condition $\eta=M_{\mathrm{i}}$, where $M_{\mathrm{i}}$ is the mobilised critical friction ratio (which may vary with stress state and void ratio). The subscript 'i' denotes the 'image condition', discussed below.

The insight from Figure 7 is that soil contacts align to best carry the imposed loads, with the contact arrangement responding to load direction as well as load magnitude. It then follows that changing the direction of load necessarily causes a softer response, and which in terms of a plasticity model might be captured by reducing the size of the yield surface - the idealisation shown in Figure 4. This idealisation is isotropic to the stress invariants but also linked to the direction of principal stress; it captures the effect of 'induced anisotropy' within an apparently isotropic yield surface.

Since critical state theory captures so much soil behaviour in a practically useful way and with rather simple models (and models that involve familiar soil properties too), it seems sensible to retain critical state theory when considering PSR. This may be achieved by adding a PSR mechanism 'on top' of the existing general theory. The general critical state theory is now briefly discussed before introducing the new features that are required to capture the effects of PSR.

\section{NorSand}

\section{Background}

NorSand is a constitutive model that was developed out of experience with hydraulic fills in the 1980s, which were originally characterised by the somewhat empirically defined state parameter $\psi$ (Been and
Jefferies, 1985, 1986). Recognition that the state parameter was fundamental to soil behaviour occurred in several places during the 1990s and there are now alternative models all using the state parameter as the measure of soil 'state' (the models differ in a few details and their authors' preferred form of the mathematical representation of the physics). All such state-based models build on critical state concepts/theory developed over the past 70 years and represent the current development of a long-established framework for understanding mechanical aspects of soil behaviour (see Jefferies and Been (2006) for a detailed historical perspective). State-based models also offer the attraction that soil properties are independent of stress level and void ratio, with the model itself computing the effect of these factors on soil behaviour. NorSand was the first of these state-based models; original Cam-Clay (Schofield and Wroth, 1968) exists as a special case of NorSand using a particular choice of soil properties and initial conditions (Jefferies and Been, 2015).

Despite the name, NorSand is simply a mathematical framework that validates across a wide range of soil types with Figure 8 showing the range so far (to the authors' knowledge, it has not been used for clay although there is no reason why it should not be). NorSand also explicitly captures the full range of soil behaviour from static liquefaction of very loose soils through to dilation of very dense soils.

Yield surface size is linked with the development of particle contacts. There is no particular difficulty with this for fixeddirection loading, but extending state-based models to include PSR must start with the basic consideration: is the critical state affected by loading direction?

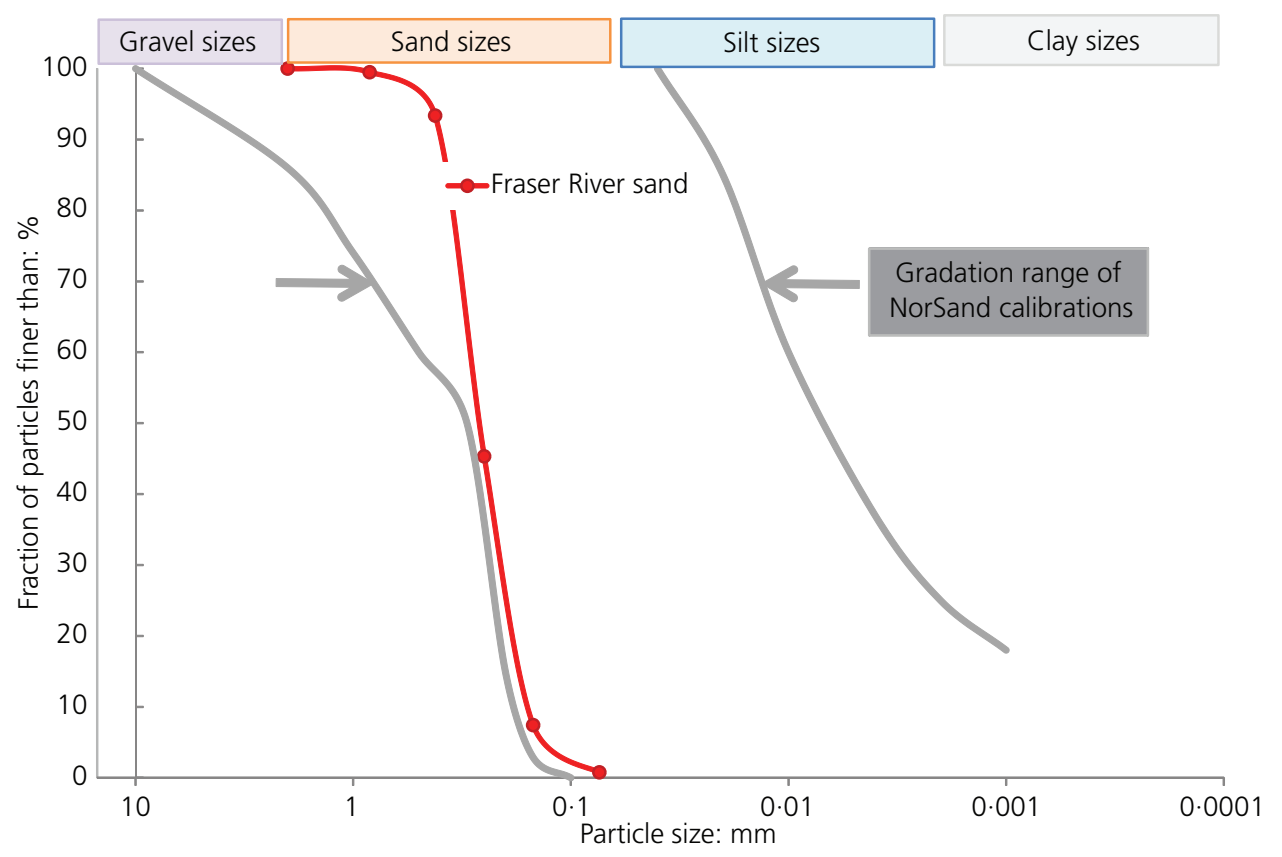

Figure 8. Range of soil gradations for NorSand calibrations to date 


\section{Critical state locus}

The starting point for the validity of critical state models is the reasonableness or otherwise of the critical void ratio, the founding idea for the framework. Critical state soil mechanics takes the critical void ratio against mean effective stress relation - universally called the critical state locus (CSL) - as the reference condition. Critical state models invoke two axioms that define the framework: (1) a unique CSL exists; (2) soils move to the CSL with shear strain.

Axiom 2, although often only appearing tacitly, is uncontroversial. There seems widespread acceptance that if a soil is sheared to sufficient shear strain, it will evolve (dilate or contract) to a constant void ratio $e_{\mathrm{c}}$, which is known as the critical state for that particular loading and initial conditions. The idea is formally written as

1. $e_{\mathrm{c}} \Leftrightarrow D^{p} \equiv 0$ and $\dot{D}^{p}=0$

where $D$ is the 'dilatancy': the ratio of volumetric strain increment to deviatoric strain increment.

Axiom 1 is still regarded as controversial by some, with confusion over what is meant by 'unique'. Mathematically, 'uniqueness' of the CSL requires no more than

2. $e_{\mathrm{c}}=C\left(\bar{\sigma}_{1}, \bar{\sigma}_{2}, \bar{\sigma}_{3}, \Phi\right)$

where $C()$ is a single-valued function (i.e. it returns only one value for $e_{\mathrm{c}}$ for any combination of the arguments within the bracket). The function $C()$ does not have to be semi-logarithmic nor only in terms of $p^{\prime}$ (the most commonly encountered form). The term $\Phi$ in the argument of $C()$ represents various state measures, which may include a fabric tensor as suggested by Li and Dafalias (2012).

The biggest difficulty over 'uniqueness' appears to be confusion over Equation 1. The critical state has to be identified from measured trends in the evolution of void ratio (or, equivalently, excess pore water pressure for an undrained test). Nearly all claims that the CSL is not unique trace back to workers who confuse what is variously called the 'phase transition' or the 'pseudo-steady' state with the critical state - those workers neglect the second condition in Equation 1. Such neglect prompted a detailed experimental investigation of the CSL by Been et al. $(1991,1992)$ which found a sensibly unique CSL, within experimental precision, for various initial fabrics and loading paths.

There is a reasonable question about how the CSL may be affected by the relative proportion of intermediate principal stress, say as characterised by the Lode angle $\theta$. The various loading paths given by Been et al. $(1991,1992)$ were all constant in direction and triaxial, and the ranges in validation paths reported by Jefferies and Shuttle (2005) were also all triaxial. Moving away from triaxial conditions is uncertain because data on the critical state in even plane strain (perhaps the most important practical situation) are sparse, with conflicting conclusions. Jefferies and Shuttle (2002) investigated the behaviour of Brasted Sand reported by Cornforth $(1961,1963)$ and concluded that $e_{\mathrm{c}}$ was unaffected by $\theta$. In contrast, tests on Changi Sand by Wanatowski and Chu (2007) showed a small but systematic shift in $e_{\mathrm{c}}$ between triaxial compression $(\theta=$ $\left.30^{\circ}\right)$ and plane strain $\left(\theta \sim 15^{\circ}\right)$ conditions; but this shift is not a case of non-uniqueness as, provided $\theta$ is included as an argument in Equation 2, there is still a single-valued prediction for $e_{\mathrm{c}}$.

This paper considers test data on FRS, and the precision by which void ratio is known in the CSS data is worse than the potential systematic shift in the CSL by the change from triaxial conditions (used to determine the CSL) and the plane strain conditions (for which soil behaviour is simulated). The practical effect of a potentially systematic change in $e_{\mathrm{c}}$ with $\theta$ is secondary to experimental accuracy in the data being considered.

The suggestion of Li and Dafalias (2012) is more troubling. If fabric affects the critical state, then the CSL will change with PSR (and possibly other paths), and much of the simplicity of a critical state framework for soil behaviour is lost. Conversely, if the critical state is a dynamic condition in which as many particle contacts are being destroyed as created - something that might be viewed as 'standard' within a micromechanical view of soil behaviour - then PSR ought to have no effect on the critical state. The idealisation adopted here is that the CSL involves its own particular fabric, with the effects of initial fabric being completely lost by the time the soil has sheared sufficiently to attain the critical state (consistent with the experimental study by Been et al. (1991)).

\section{NorSand for fixed principal stress direction}

NorSand is a work-hardening plasticity model derived from the axioms of critical state theory and with an idealised work dissipation mechanism. As such, the key aspects are (i) the yield surface, (ii) the 'flowrule' giving relative plastic strain increments and (iii) the hardening law, which controls how the yield surface responds to plastic strain.

The NorSand yield surface has the familiar bullet-like shape of the original Cam-Clay model but with one important difference - there is an internal cap so that the soil cannot unload to very low mean stress without yielding. This internal cap is taken as a flat plane, and its location depends on the soil's current state parameter. Figure 9 illustrates the NorSand yield surface for two cases: a very loose soil and a very dense soil. Also illustrated in Figure 9 is the location of the internal cap which controls the limiting stress ratio $\eta_{\mathrm{L}}$ that the soil can sustain; what is sometimes called the 'Hvorslev Surface' is simply the locus of hardening limits for a properly derived yield surface. This internal cap is no more than a strict implementation of the ideas of Drucker et al. (1957) and gives realistic dilatancy with normality.

The plastic dilatancy $D^{p}$ is determined from the idealised stressdilatancy relationship that underlies the model, but as there are three strain rates, stress-dilatancy alone is insufficient to determine each of these strain rates. The intermediate principal 
Principal stress rotation as cause of

cyclic mobility

Jefferies, Shuttle and Been

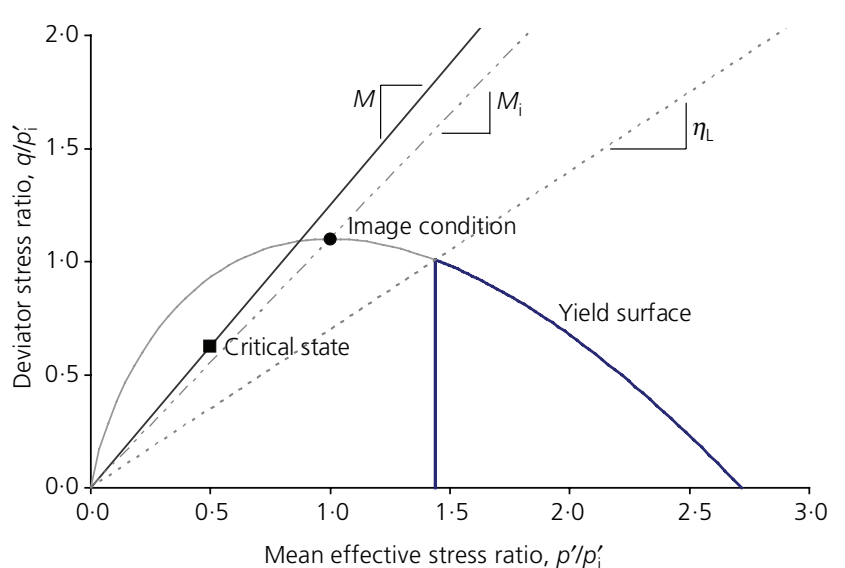

(a)

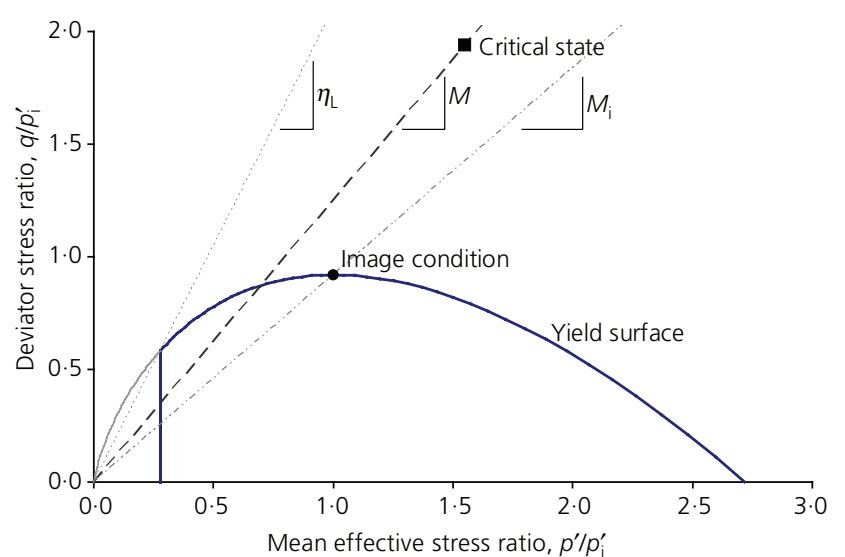

(b)

Figure 9. Illustration of NorSand yield surfaces and limiting stress ratios; (a) very loose sand, (b) very dense sand

strain rate is therefore interpolated depending on the Lode angle. This interpolation approach is somewhat unusual for plasticity models but is taken to ensure consistency with the work dissipation postulate that is the basis of the model (Jefferies and Shuttle, 2002).

NorSand adopts isotropic hardening, with the yield surface expanding or shrinking while retaining its shape. The size of the yield surface is scaled by the image stress $\bar{\sigma}_{\mathrm{m}, \mathrm{i}}$, which forms the object of the hardening law. It is called the image stress because it represents a situation in which one of the two conditions for the critical state is met, as illustrated in Figure 9. The hardening law was derived by explicitly invoking axiom $2: \psi \rightarrow 0$ as $\varepsilon_{q} \rightarrow \infty$. In some ways, the NorSand hardening law could be viewed as going back to Casagrande's original experiments that produced the concept of critical void ratio, those experiments elegantly illustrating that the critical void ratio is an end-state and not something that should anchor yield surfaces throughout the loading path (as is done in both variants of Cam-Clay). Thus, as shown in Figure 9, the critical state does not usually intersect the yield surface (this is the largest single difference between NorSand and Cam-Clay). This divergence of yield surface from critical state is used as the basis of the hardening law, the hardening law acting to move the yield surface towards the critical state under the action of plastic shear strain - directly capturing the essence of critical state principles.

NorSand was originally formulated for triaxial compression (Jefferies, 1993), as is usual, with subsequent generalisation to three dimensions (Jefferies and Shuttle, 2002). The representation of the critical friction ratio was subsequently simplified by Jefferies and Shuttle (2011). The equations for NorSand are summarised in Table 1.

The soil properties used in NorSand are largely familiar, with some flexibility depending on the level of detail sought. All properties are dimensionless and determined by triaxial compression tests. In the simplest form, three properties $\left(M_{\mathrm{tc}}, \Gamma, \lambda\right)$ are used to define the critical state; a slightly more detailed idealisation for the critical state of FRS is used here involving a 'curved' CSL (discussed when presenting the test data). Two properties are associated with strength at peak dilatancy: $N$ the volumetric coupling following Nova (1982) and $\chi$ determining the influence of $\psi$ on maximum dilatancy. The plastic hardening modulus, $H$, is needed because the yield surface is now decoupled from the CSL, although there is an approximate proportionality of $H \sim 4 / \lambda_{\mathrm{e}}$ echoing aspects of the Cam-Clay variants. In terms of elasticity, the simplest approach uses just two properties, $I_{\mathrm{r}}$ and $v$; a more sophisticated elastic idealisation is used for FRS, described when presenting the data for this sand. Table 2 summarises NorSand properties together with comments on their numerical values for various soils.

Calibration procedures are presented in the paper by Jefferies and Shuttle (2005) with further details in the paper by Jefferies and Been (2006). Only standard triaxial tests found in normal engineering practice are needed, as would be expected from the use of widely adopted soil properties (i.e. most of the properties listed in Table 2 are not specific to NorSand). Calibration is illustrated later for the tested FRS.

\section{Including PSR in NorSand}

The principle used to include PSR in NorSand is taken directly from the 'induced anisotropy' of particle contacts seen in micromechanical studies. Formation of particle contacts with plastic strain is captured, in plasticity models, by change in the stress(es) that scale the yield surface - in the case of NorSand, this is just $\bar{\sigma}_{\text {mi }}$. So reduction in the proportion of appropriately oriented contacts because of PSR amounts to annealing of the previously established hardening of the yield surface - the yield surface must shrink as the loading changes direction. The original hardening law is then modified to capture PSR

3.

$$
\frac{\dot{\bar{\sigma}}_{\mathrm{mi}}}{\bar{\sigma}_{\mathrm{mi}}}=\left[\frac{d \bar{\sigma}_{\mathrm{mi}}}{d \varepsilon_{q}^{p}}\right] \dot{\varepsilon}_{q}^{p}+\left[\frac{\dot{\bar{\sigma}}_{\mathrm{mi}}}{\bar{\sigma}_{\mathrm{mi}}}\right]_{\mathrm{PSR}}
$$

where the first term on the right-hand side of Equation 3 is the normal fixed-direction hardening (i.e. the existing model as used for triaxial calibration and so forth) with the second term then being 
Internal model variables

Critical state

Yield surface and internal cap $\left(\bar{\sigma}_{\mathrm{mi}}=\bar{\sigma}_{\mathrm{mx}}\right)$

Stress dilatancy

Hardening rule

Plastic strain increment ratios (these strain rate ratios are consistent with stress dilatancy equation for all stress states, see Jefferies and Shuttle (2002))

Elasticity $\psi_{\mathrm{i}}=\psi+\lambda \ln \left(\bar{\sigma}_{\mathrm{mi}} / \bar{\sigma}_{\mathrm{m}}\right)$ where $\psi=\mathrm{e}-\mathrm{e}_{\mathrm{c}}$

$M_{\mathrm{i}}=M\left(1-N \chi_{\mathrm{i}}\left|\psi_{\mathrm{i}}\right| / M_{\mathrm{tc}}\right)$

$e_{\mathrm{c}}=\Gamma-\lambda \ln \left(\bar{\sigma}_{\mathrm{m}}\right)$ and $\eta_{\mathrm{c}}=M=M_{\mathrm{tc}}-\frac{M_{\mathrm{tc}}^{2}}{3+M_{\mathrm{tc}}} \cos \left(\frac{3 \theta}{2}+\frac{\pi}{4}\right)$

$\frac{\eta}{M_{\mathrm{i}}}=1-\ln \left(\frac{\bar{\sigma}_{\mathrm{m}}}{\bar{\sigma}_{\mathrm{mi}}}\right)$ with $\left(\frac{\bar{\sigma}_{\mathrm{mx}}}{\bar{\sigma}_{\mathrm{m}}}\right)=\exp \left(-\chi_{\mathrm{tc}} \psi_{\mathrm{i}} / M_{\mathrm{i}, \mathrm{tc}}\right)$

$D^{p}=M_{i}-\eta$

On outer yield surface:

$\frac{\dot{\bar{\sigma}}_{\mathrm{mi}}}{\bar{\sigma}_{\mathrm{mi}}}=H \frac{M_{\mathrm{i}}}{M_{\mathrm{i}, \mathrm{tc}}}\left(\frac{\bar{\sigma}_{\mathrm{m}}}{\bar{\sigma}_{\mathrm{mi}}}\right)^{2}\left[\frac{\bar{\sigma}_{\mathrm{mx}}}{\bar{\sigma}_{\mathrm{m}}}-\frac{\bar{\sigma}_{\mathrm{mi}}}{\bar{\sigma}_{\mathrm{m}}}\right] \dot{\varepsilon}_{q}^{p}+S \frac{\eta}{\eta_{\llcorner}} \frac{\dot{\bar{\sigma}}_{\mathrm{mx}}}{\bar{\sigma}_{\mathrm{mx}}}$

...where $S=0$ or 1 (a Boolean switch) and $\eta_{\mathrm{L}}=M_{\mathrm{i}}\left(1-\chi_{\mathrm{i}} \psi_{\mathrm{i}} / \mathrm{M}_{\mathrm{i}, \mathrm{tc}}\right)$

On internal cap:

$\frac{\dot{\bar{\sigma}}_{\mathrm{mi}}}{\bar{\sigma}_{\mathrm{mi}}}=-\frac{H}{2} \frac{M_{\mathrm{i}}}{M_{\mathrm{i}, \mathrm{tc}}}\left|\dot{\varepsilon}_{q}^{p}\right|$

define $D_{\mathrm{tc}}^{p}=D^{p} M_{\mathrm{i}, \mathrm{tc}} / M_{\mathrm{i}}$ and $D_{\mathrm{te}}^{p}=D^{p} M_{\mathrm{i}, \mathrm{te}} / M_{\mathrm{i}}$

define $z_{3, \mathrm{tc}}=\frac{2 D_{\mathrm{tc}}^{p}-3}{6+2 D_{\mathrm{tc}}^{p}}$ and $z_{3, \mathrm{tc}}=\frac{2 D_{\mathrm{te}}^{p}-6}{3+2 D_{\mathrm{te}}^{p}}$

$\frac{\dot{\varepsilon}_{3}^{p}}{\dot{\varepsilon}_{1}^{p}}=z_{3}=z_{3, \mathrm{tc}}-\left(z_{3, \mathrm{tc}}-z_{3, \mathrm{te}}\right) \cos \left(\frac{3 \theta+90}{2}\right)$

define $a=(\sin \theta+\sqrt{3} \cos \theta) / 3, b=-2 \sin \theta / 3, c=(\sin \theta-\sqrt{3} \cos \theta) / 3$

$\frac{\dot{\varepsilon}_{2}^{p}}{\dot{\varepsilon}_{1}^{p}}=z_{2}=\left(a D^{p}-1+\frac{\dot{\varepsilon}_{3}}{\dot{\varepsilon}_{1}}\left(c D^{p}-1\right)\right) /\left(1-b D^{p}\right)$

$I_{\mathrm{r}}=\frac{G}{\bar{\sigma}_{\mathrm{m}}}$ with $K=\frac{2(1+v)}{3(1-2 v)} G \ldots$ normally use $I_{\mathrm{r}}$ as constant but other relations for $G$ admissible provided that $v=$ constant

Table 1. Summary of monotonic NorSand 


\begin{tabular}{|c|c|c|c|}
\hline & Fraser River sand & Typical range & Comment \\
\hline \multicolumn{4}{|l|}{ CSL } \\
\hline$\Gamma$ & $1 \cdot 22$ & $0 \cdot 9-1 \cdot 4$ & 'Altitude' of CSL, defined at $1 \mathrm{kPa}$ \\
\hline$\lambda_{10}$ & $0 \cdot 138$ & $0 \cdot 03-0 \cdot 15$ & $\begin{array}{l}\text { Slope of CSL, defined on base } 10 \\
\text { See paper text for curved CSL }\end{array}$ \\
\hline \multicolumn{4}{|c|}{ Plasticity } \\
\hline$M_{\mathrm{tc}}$ & $1 \cdot 45$ & $1 \cdot 2-1 \cdot 5$ & Critical friction ratio, triaxial compression as reference condition \\
\hline$N$ & 0.43 & $0 \cdot 2-0.5$ & Volumetric coupling parameter \\
\hline$\chi_{\text {tc }}$ & $3 \cdot 40$ & $2 \cdot 5-4 \cdot 5$ & $\begin{array}{l}\text { Relates minimum dilatancy to corresponding } \psi \text {, triaxial as } \\
\text { reference condition }\end{array}$ \\
\hline$H$ & $60-280 \psi$ & $50-500$ & Plastic hardening modulus for loading, often $\mathrm{f}(\psi)$ \\
\hline$Z$ & $\sim 10$ & n/a & Plastic softening modulus for principal stress rotation \\
\hline \multicolumn{4}{|c|}{ Elasticity } \\
\hline Ir & $450-750$ & $100-800$ & $\begin{array}{l}\text { Dimensionless shear rigidity } \\
\text { See paper text for adopted relation }\end{array}$ \\
\hline$v$ & $0 \cdot 1$ & $0 \cdot 1-0 \cdot 3$ & Poisson's ratio, not usually measured \\
\hline
\end{tabular}

the additional softening to capture the effects of PSR. A simple idealisation for this second term is

4. $\left[\frac{\dot{\bar{\sigma}}_{\mathrm{mi}}}{\bar{\sigma}_{\mathrm{mi}}}\right]_{\mathrm{PSR}}=-Z\left|\frac{\dot{\alpha}}{\pi}\right|$

where $Z$ is a soil property. The negative sign and modulus function enforce softening under PSR. The scaling factor of $\pi$ radians makes Equation 4 dimensionless (if working with degrees, this scaling factor becomes 180). But, there are limitations on this simple idea.

First, the reduction in yield surface hardness can only go so far as there is a minimum yield surface size for the current mean effective stress (corresponding to no induced anisotropy)

5. $\quad \bar{\sigma}_{\mathrm{mi}}>\bar{\sigma}_{\mathrm{m}} / r$

where $r$ is the yield surface spacing ratio $(r=2.71$ for the NorSand version used here). This restriction of Equation 5 on softening is implemented by modifying Equation 4 such that PSR only affects induced anisotropy

6. $\left[\frac{\dot{\bar{\sigma}}_{\mathrm{mi}}}{\bar{\sigma}_{\mathrm{mi}}}\right]_{\mathrm{PSR}}=-Z\left(\frac{\bar{\sigma}_{\mathrm{mi}}}{\bar{\sigma}_{\mathrm{m}}}-\frac{1}{r}\right)\left|\frac{\dot{\alpha}}{\pi}\right|+\frac{1}{r}$

Second, if the critical state is a dynamic condition in which as many particle contacts are being destroyed as created (as discussed earlier), the PSR must have no effect at the critical state. Such a further restriction on the effects of PSR allows axiom 2 to continue in its original form derived from Casagrande's idealisation. An elegant way of doing this is to modify Equation 6 further by scaling the reduction using the state parameter

7. $\left[\frac{\dot{\bar{\sigma}}_{\mathrm{mi}}}{\bar{\sigma}_{\mathrm{mi}}}\right]_{\mathrm{PSR}}=\left[-Z\left(\frac{\bar{\sigma}_{\mathrm{mi}}}{\bar{\sigma}_{\mathrm{m}}}-\frac{1}{r}\right)\left|\frac{\dot{\alpha}}{\pi}\right|+\frac{1}{r}\right] \mid \psi_{\mathrm{i}}$

where $\psi_{\mathrm{i}}$ evolves throughout the test. Of course, it could be argued that there might be non-linear scaling of rotation effects (e.g., a cosine function) and that Equation 7 is simplistic, with experimental data needed to guide choices. But a first approach should always be to use the simplest rule that is physically plausible and consistent with the idealisations of the theory - and that approach is followed here. The validity of Equation 7 derives from how well it works in simulating test data, which is shown later in this paper.

A more subtle consequence of PSR is that at least temporary noncoincidence must arise between the directions of principal strain increments and principal stresses. Gutierrez and Ishihara (2000) consider this non-coincidence and an adaption of their work would be to modify the stress-dilatancy relation to

8. $D^{p}=M_{\mathrm{i}}-\eta \cos (\beta)$

where $\beta$ is the lag angle between the $\sigma_{1}$ direction associated with the current yield surface and the $\sigma_{1}$ direction in situ (see Figure 4). This then leads to a further consideration: how should $\beta$ vary? 
Clearly, if there is a step rotation of $\sigma_{1}$ to a new direction and shearing then continues with the constant new direction, at some time coincidence of stress and strain increment will be restored (at least for self-consistency of the idealised model if not in complete reality of soil behaviour). A viscous annealing function for $\beta$ would seem appropriate, but doing this adds complexity and yet more soil properties.

A goal of this paper is to illustrate how a very simple idea captures much of the observed soil behaviour and, consistent with the development of critical state theory, it is reasonable to trade some accuracy for consistency and simplicity. Hence $\beta=0$ is adopted as well as instantaneous rotation of the yield surface with $\sigma_{1}$. These assumptions should be reasonable during continuous rotation of $\sigma_{1}$. Equally, the assumptions can be expected to be deficient near each change of loading direction.

\section{Numerical integration}

NorSand has no closed form solution, even for standard laboratory tests where the stress path is known - numerical integration is needed. In the case of the fixed principal stress direction (e.g. the triaxial test), numerical integration is simply done using Euler's method with small steps of the plastic shear strain $\varepsilon_{q}$ to drive the hardening law. The stress state is then solved by means of the consistency condition, this condition following from the equations of the yield surface in the usual manner, which is

9.

$$
\frac{\dot{\eta}}{M}=\left(\frac{\dot{\bar{\sigma}}_{\mathrm{mi}}}{\bar{\sigma}_{\mathrm{mi}}}-\frac{\dot{\bar{\sigma}}_{\mathrm{m}}}{\bar{\sigma}_{\mathrm{m}}}\right)
$$

Further details on the integration procedure can be found in the paper by Jefferies and Been (2006) and the integration is easily implemented in a spreadsheet, so allowing convenient comparison of theory and data. In practice, small step sizes are not an issue and Euler's method is more than adequate for the purpose with the added advantage of being easy to understand. The step size needed depends on the elastic stiffness; the downloadable spreadsheet uses steps approximately equivalent to elastic changes in the deviator stress of $\Delta q=1 \mathrm{kPa}$, but this has not been optimised (step sizes can readily be changed in the VBA code).

In the case of PSR, the numerical issue is whether to integrate (solve) Equation 3 in a single Euler step - which may need something like substepping with the bisection algorithm since the fixed-direction stress change affects the PSR-induced softening or whether to use a two-step approach with initial fixed-direction hardening being followed to derive the new stress state and with the PSR-induced softening then re-setting the yield surface hardness for the next step. Numerically, Euler integration necessarily involves small steps and little is given up in terms of accuracy with a two-step approach. Further, a two-step integration allows direct use of the verified and validated three-dimensional (3D) fixed-direction algorithm; only a little extra code is added to cope with simulating PSR. The results reported here used the two-step integration of Equation 3.

It is possible to have elastic as well as plastic loading in simple shear (whether monotonic or cyclic). The easiest algorithm to use is an elastic prediction of the stress change resulting from imposed displacement, which is then updated with a plastic 'corrector' capturing the proportion of the imposed displacement that is plastic. This is a standard numerical approach and detailed derivations for NorSand are provided as a downloadable supplement.

A simplification in the numerical integration for CSS is that the loading is applied in constant displacement steps until reaching the target stress for motion-reversal. This differs from laboratory equipment that usually offers servo-controlled sinusoidal stress histories. However, there are no rate-terms in the constitutive equations so the actual shape of the load-time history does not affect the accumulation of strain or pore pressure with load cycling. It is only a consideration of how to map the computed stress to the elapsed time. Here the numerical complexity of adding the equivalent of a load control servo to the integration is avoided in favour of the computationally easier alignment of simulation with peaks in cyclic shear stress of the data using a (fractional) cycle counter.

Some purely elastic PSR are possible - for example, the start of the load reversal during a CSS test. Elastic PSR also leads to the situation where there can be jumps in the principal stress direction, most notoriously arising in the cyclic triaxial test with $\sigma_{1}$ flipping between axial and radial directions during load reversal. Thus, Equation 7 may be applied continuously during loading or as a 'largish' change in yield surface size during a jump change in the principal stress direction. This is not a conceptual issue and only requires care in programming to capture the possibility of sudden reduction in yield surface size at the end of an elastic unloading.

The implementation of these ideas is provided as commented open-source VBA code within the subroutine $\operatorname{NorCSSu()}$ of the downloadable spreadsheet.

\section{Example of computed soil behaviour in CSS test}

At this point, it is helpful to have an example illustrating what Equation 7 delivers in terms of computed soil response, highlighting aspects that are well-captured as well as aspects that are missed. The effects of soil properties on the computed response are then illustrated. Subsequently, the calibration to a particular data set is developed, leading to discussion of the overall performance of the PSR-induced annealing across a range of void ratios and cyclic stress levels, and including 'static bias'.

The example is shown in Figure 10 and comprises a lightly dilatant sand $\left(\psi_{0} \sim-0 \cdot 1\right)$ loaded with a cyclic stress amplitude CSR $=0 \cdot 1$ (a mid-range value for this type of test on compact sand) and without any static bias; the tested sample 'failed' after six load cycles using the 'standard' criterion that $\mathrm{N}_{\mathrm{L}} \Leftrightarrow \gamma_{\mathrm{xy}}>3.75 \%$, a convenient 

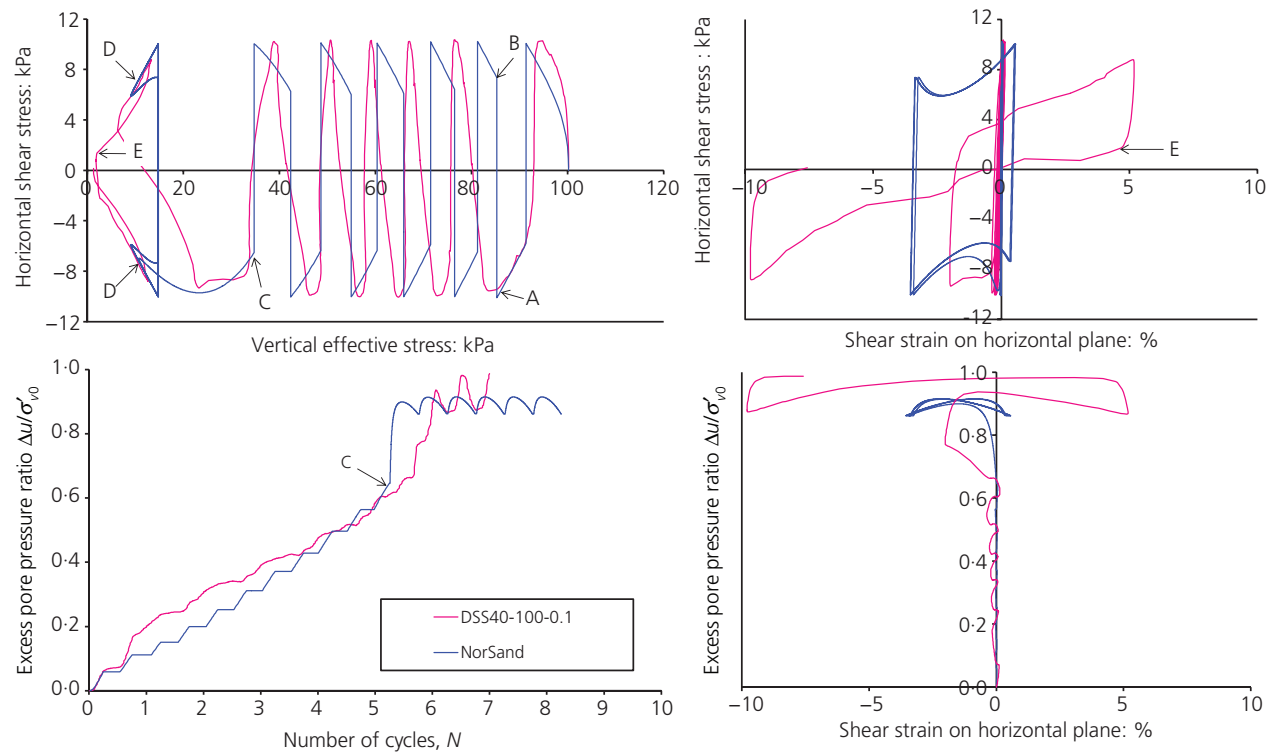

Figure 10. Example of computed soil response during PSR

example allowing sufficient cycles to illustrate the main features but without becoming too cluttered to see the details. Nevertheless, because of the many changes in stress path direction, this figure is best viewed in colour (as per the pdf file) to distinguish between the measured soil response (magenta) from that computed (in blue).

As can be seen in Figure 10, the computed stress path is a good match to the measured data for the first positive and subsequent negative loading cycle. But, then at the annotated point A, the computed model unloads elastically and partly reloads elastically, before yielding restarts at point $\mathrm{B}$; whereas the measured sand behaviour shows continuous yielding (positive excess pore pressure rate, with the vertical effective stress continuously decreasing). The computed behaviour continues as a sequence of elastic (vertical stress path) and plastic (curved stress paths) phases in contrast to the measured data which indicated continuous yielding; nevertheless, the overall rate of increase in excess pore pressure with load cycle is comparable, as can be seen in Figure 10, and with the same sudden increase in the trend once $r_{\mathrm{u}}>\sim 0.65$ indicated by point $\mathrm{C}$. This sudden increase in excess pore pressure leads to the next stage in the evolution of the sand behaviour when the stress ratio $\eta>M_{\mathrm{i}}$, readily seen in the data as a transition into dilation as indicated by point D. Dilation produces negative excess pore pressure and the evolution of the computed response enters a steady cyclic situation where the negative excess pore pressure during the later dilating part of the load cycle exactly balances the positive pore pressure caused by the annealed response during the earlier part of the loading cycle. Where the soil response stabilises depends on the state-dilatancy (controlled by the state parameter and the soil property $\chi$ ) and the annealing caused by PSR (Equation 7 and the soil modulus Z). In the case of Figure 10, this stabilisaion of computed soil response is at $r_{\mathrm{u}}$ $\sim 0.9$ which is less than, even transient, 'full liquefaction'.
A further aspect of the behaviour is the computed PSR, shown in Figure 11, which corresponds to the stress-strain results shown in Figure 10. Although the first two loading cycles show smooth PSR, once yielding becomes established the situation transitions into a square-wave of principal stress flips between the limiting
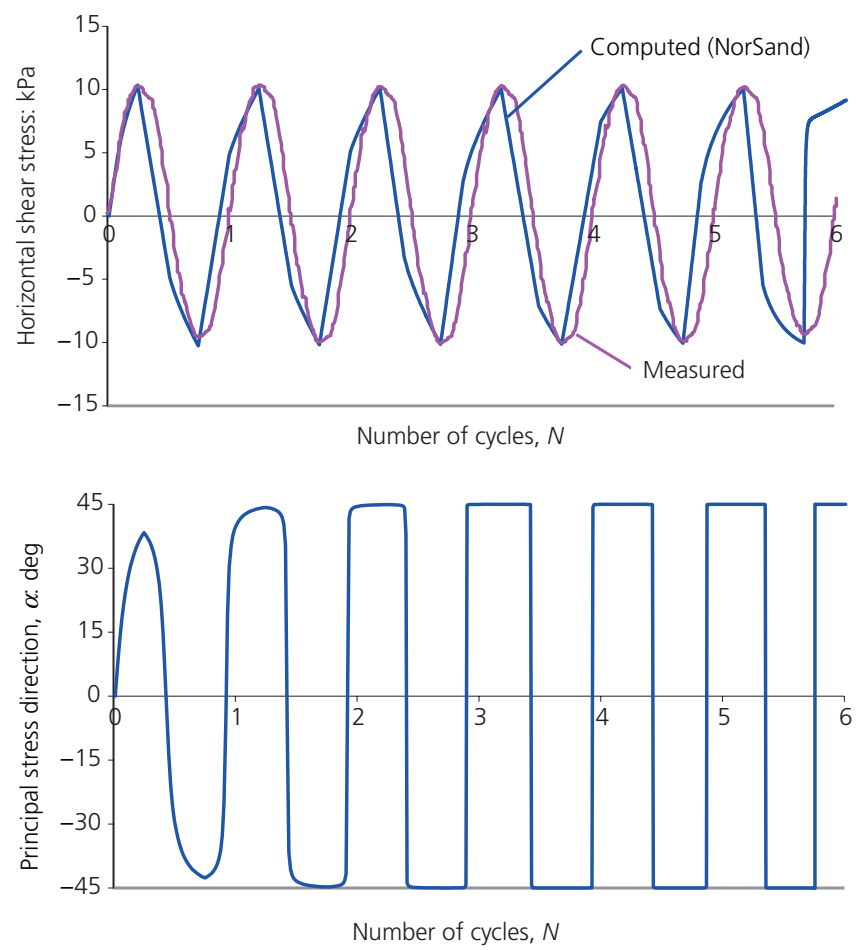

Figure 11. Effect of plasticity on PSR 
$45^{\circ}$ situations of positive and negative loading. The peak stress is replicated, but there is not a perfect simulation of the load-time history because the VBA integration used was 'constant strain steps then reverse' rather than using a servo routine (as commented on earlier).

In terms of shear strain, the computed response in Figure 10 is similar to that measured with only small strains until the soil first encounters point $\mathrm{D}$, whereupon large strains arise. However, there is a difference in character at this point between model and data. The model computes elastic unloading, followed by substantial annealing on the principal stress flip, and then extreme dilation (the soil is moving to ever more dilatant values of $\psi$ because of the decreasing mean effective stress at constant void ratio); the corresponding strain hysteresis loop has a 'square' wave character. In contrast, the measured data show apparently elastic strains in initial 'unloading' but with decreasing mean effective stress that would be associated with plastic yielding, which are then followed by extreme loss of stiffness (true yield) just before the principal stress flip, indicated as point $\mathrm{E}$ in Figure 10.

\section{Effect of softening modulus Z}

A single new soil property is added to NorSand to simulate the effect of cyclic loading: the softening modulus $Z$ that proportions the amount of PSR-induced annealing. The effect of this property on the computed soil response is illustrated in Figure 12(a), which shows a change in the approximately linear rate of excess pore pressure increase until dilation sets in. What is less obvious from this figure is that the maximum excess pore pressure also depends on $Z$, with $Z=6 \rightarrow r_{\mathrm{u}}=0.88$ while $Z=18 \rightarrow r_{\mathrm{u}}=0.92$; there is a corresponding change in the final cyclic strain amplitude.

The rate of increase in excess pore pressure does not depend only on $Z$, with the soil's usual stress-strain response under fixed principal stress direction also affecting the cyclic behaviour. This develops because a substantial part of the imposed load cycle in the CSS test is actually at fixed principal stress direction $\left( \pm 45^{\circ}\right)$, as can be seen in Figure 11. The effect of the soil property $H$ on the rate of excess pore pressure development is illustrated in Figure 12(b) for a constant annealing modulus $Z=12$; there is a similar effect of change in $H$ to change in $Z$. A corollary of Figure 12 is that the intrinsic soil properties associated with fixed principal stress direction must be known before developing a characterisation for the effect of cyclic loading from the CSS test - there are competing effects and these must be isolated. A series of CSS tests alone will be insufficient to characterise soil.

\section{FRS}

With the understanding that all the soil properties affect the soil's response in cyclic loading, it is now necessary to show the determination of those soil properties for the FRS used in extensive CSS testing. Following property determination, the pattern of computed fits to this cyclic data can be developed (the previously shown Figure 10 being one of those tests).

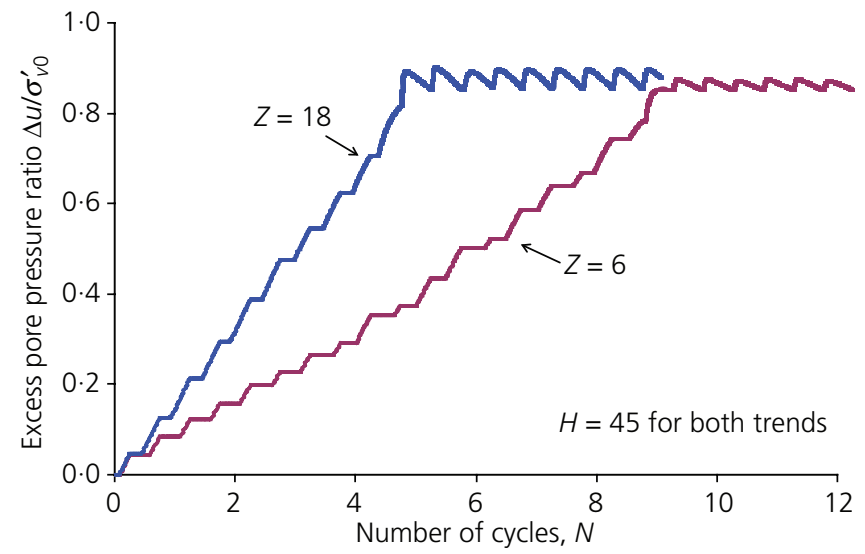

(a)

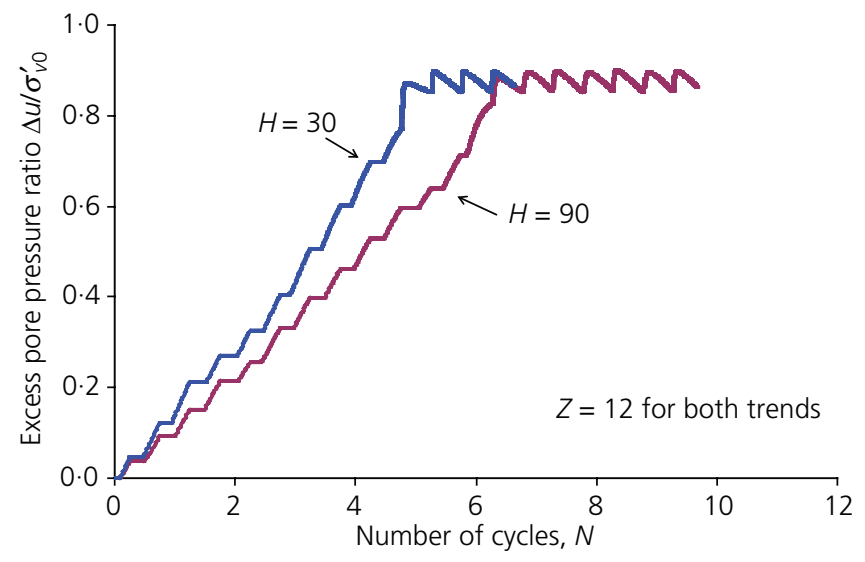

(b)

Figure 12. Effect of plastic softening modulus on rate of excess pore pressure development for otherwise constant soil properties. (a) Constant plastic modulus, (b) constant PSR softening modulus

\section{Sand tested}

FRS is an alluvial deposit widespread in the Fraser River Delta of the lower mainland of British Columbia, Canada. The area includes the city of Vancouver and is of considerable economic importance. Lying on the west coast of North America, the area is vulnerable to earthquakes and the FRS deposits are known to have liquefied in past earthquakes (e.g. from relic sand boils found in excavations).

Given the wide distribution of FRS in the highly populated and seismically active area near Vancouver, FRS has been quite well researched including contributions from Wride and Robertson (1997), Chillarige et al. (1997), Konrad (1997), Sukumaran and Ashmawy (2002) and Ghafghazi and Shuttle (2010), among others. However, despite the number of contributions in the literature, the properties of FRS are not well defined because, being a natural sand with varying proportions of fines, there is significant natural variation geographically and different workers have tested FRS from different sources.

A representative (or 'standard') example of FRS was used for both the triaxial and CSS tests reported here. The gradation tested contains around $0 \cdot 8 \%$ 'fines' (<\#200 sieve) and had $D_{50}$ and $D_{10}$ of $0 \cdot 271 \mathrm{~mm}$ 
Geotechnical Research

Volume 2 Issue 2
Principal stress rotation as cause of

cyclic mobility

Jefferies, Shuttle and Been and $0.161 \mathrm{~mm}$, respectively. FRS is a uniform, angular to subangular, with low to medium sphericity (Figure 13), medium grained clean sand with $e_{\min }=0.62, e_{\max }=0.94$ (Sriskandakumar, 2004) and $G_{\mathrm{s}}=$ 2.719 (Shozen, 1991). The average mineral composition based on a petrographic examination is $25 \%$ quartz, $19 \%$ feldspar, $35 \%$ metamorphic rocks, $16 \%$ granites and $5 \%$ miscellaneous detritus. The gradation of the tested FRS is shown in Figure 8, allowing comparison to other soils for which NorSand has been validated.

\section{Elasticity (soil property $G_{\max }$ )}

The elastic shear modulus was determined from bender element data. These data were fitted with an elastic model that accounts for the effect of confining stress and void ratio. Pestana and Whittle (1995) reviewed acceptable and unacceptable elastic models from the perspective of applied mechanics and considering soil porosity, but a further step of working with void ratio rather than porosity

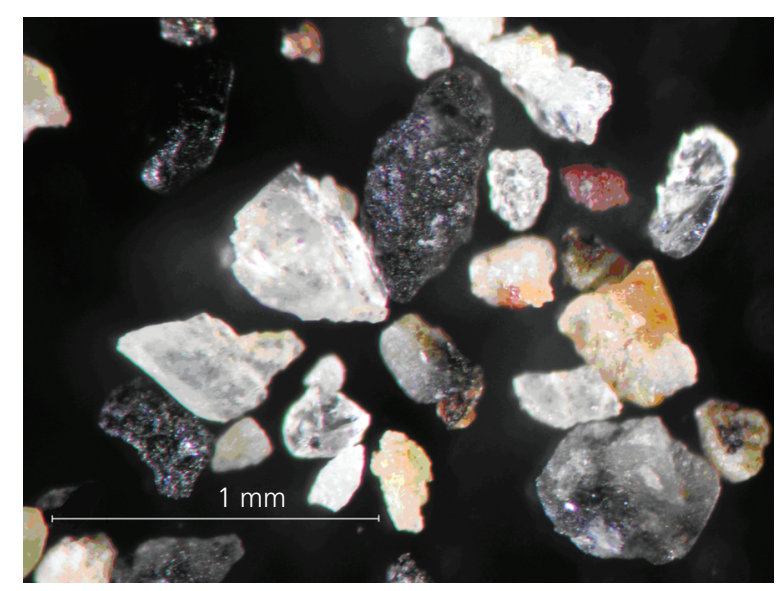

Figure 13. Microscopic picture of FRS grains tends to fit sands better (Jefferies and Been, 2000). The model adopted here complies with the acceptable forms identified by Pestana and Whittle and is

10. $G_{\max }=\frac{A \sigma_{\text {ref }}}{\left(e-e_{\text {min }}\right)}\left(\bar{\sigma}_{\mathrm{m}} / \sigma_{\text {ref }}\right)^{b}$

where $G_{\max }$ is the true elastic shear modulus (the 'max' subscript comes from current usage in geotechnical practice although strictly unnecessary to denote elasticity) and $A, e_{\min }$ and $b$ are the three soil properties of this model; by convention $\sigma_{\text {ref }}$ is $100 \mathrm{kPa}$. The model amounts to a power law dependence on confining stress consistent with contacts between soil particles not being that of ideal spheres, and with the further idea that as the void ratio tends to the soil transitioning into a sedimentary rock (at $\left.e_{\text {min }}\right)$, so the compressibility tends to zero. In practice, it is found that $e_{\min }$ in Equation 10 is slightly less (i.e. denser) than the value determined using the ASTM D4254 procedure (ASTM, 2000).

The elastic soil properties were determined by unweighted multivariate optimisation across the entire set of elastic data on FRS and gave $A=375, e_{\min }=0.344$ and $b=0.466$. The fit of the elastic model, using these soil properties, to the measured data is shown in Figure 14. Most of the data lie within 5\% of the elastic idealisation and without apparent bias.

Poisson's ratio was not measured; a value of $0 \cdot 15$ was adopted for modelling as 'not unreasonable'.

\section{Triaxial test methodology}

Triaxial testing comprised nine drained and seven undrained compression tests on samples prepared using the moist tamping technique; the state paths for these tests are shown in Figure 15. All

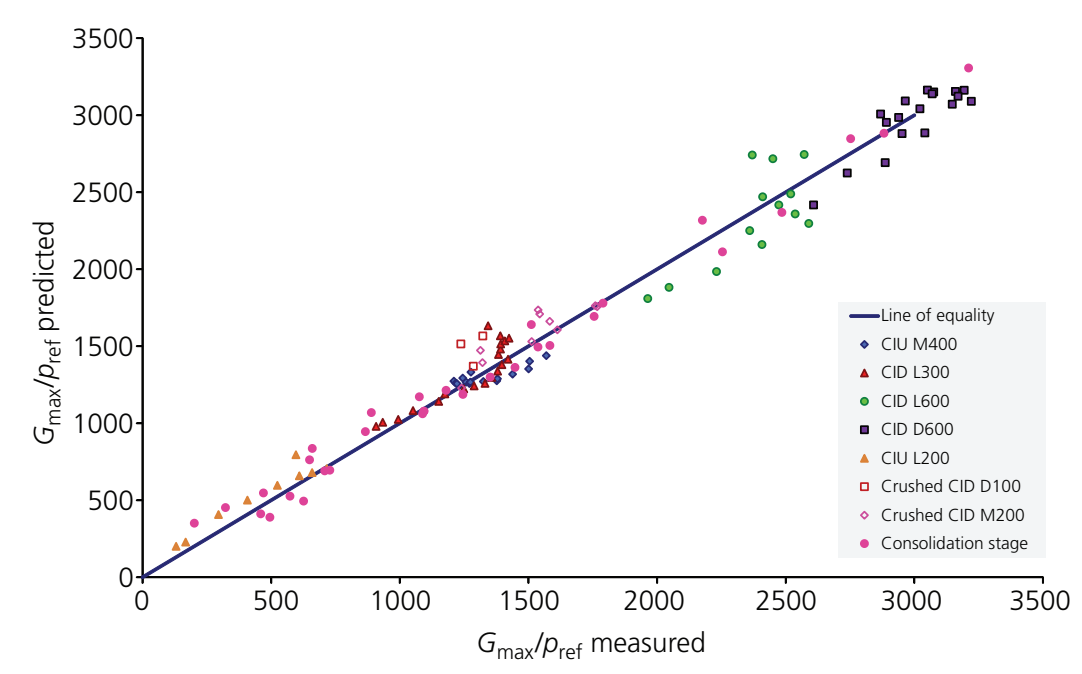

Figure 14. Comparison between measured and modelled FRS elasticity 


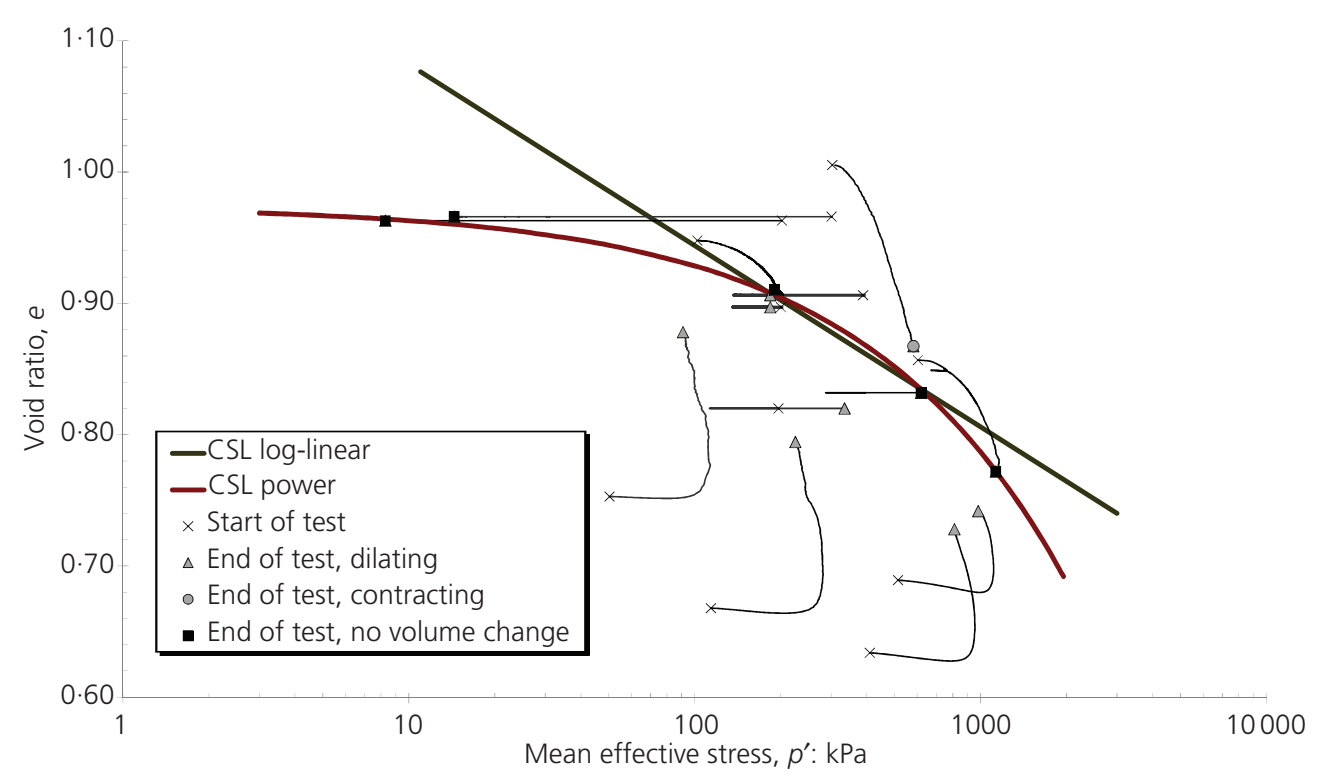

Figure 15. State diagram of triaxial tests with power law CSL

samples were $142 \mathrm{~mm}$ in height and $71 \mathrm{~mm}$ in diameter. Lubricated end platens were used, with the samples back-pressured until a $B$ value of 0.95 or greater, with samples consolidated and then sheared using controlled displacement at a constant rate of 5\% per hour. Samples were sheared until a steady state was reached, apparent shear localisation was observed, or the equipment limitations were met.

At the end of the shearing phase, the drainage valves were closed. The sample was then removed from the cell and the cell base, membrane and cap were dried externally before putting the retained sample in a freezer. The frozen sample was then extracted carefully to ensure no water or grains were lost during the process. This technique effectively eliminated loss of water during sample extraction, enabling accurate determination of water contents. A repeatability of 0.01 or better was obtained for three pairs of tests that were targeted to start from identical conditions.

During data reduction, corrections were applied for membrane penetration (Vaid and Negussey, 1982) and membrane force (Kuerbis and Vaid, 1988).

\section{CSL (soil properties $\Gamma, \lambda_{10}$ )}

An example of the behaviour of loose FRS in undrained triaxial compression is shown in Figure 16; a clear critical state is evident (although interestingly the post-peak softening is softer than commonly seen with standard quartz sands, which is consistent with the rather compressible behaviour of FRS). Not all undrained tests were equally loose, with several tests showing ' $\mathrm{S}$ '-shaped stress paths; the end points of these loose tests are marked on the state paths in Figure 15. Loose drained tests also provide useful data on the critical state, although few such tests actually reach the critical state within the strain limits of triaxial equipment; the critical state lies a little denser than the end point of such loose drained paths.
As is usual, the state paths for dense tests move towards the critical state but such dense tests are well removed from critical conditions within the strain limits of the triaxial test.

These data have been used to estimate the CSL shown in Figure 15. A conventional semi-log idealisation of the CSL can be fitted to the data using the properties $\Gamma=1 \cdot 22, \lambda_{10}=0 \cdot 138$; the slope of the CSL as measured by $\lambda_{10}$ is three to four times larger than what is generally reported for standard laboratory quartz 'reference' sands (e.g. Jefferies and Been, 2006), indicating that FRS is markedly more compressible than much testing experience. It is also evident from Figure 15 that a semi-log trend is not a particularly good representation of the CSL with an improved CSL being given by the power-law idealisation shown in Figure 15

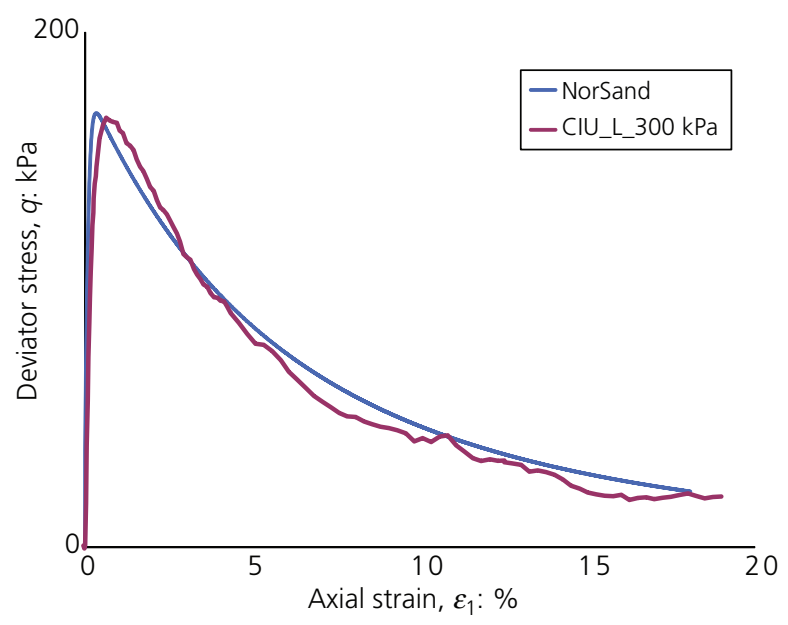

Figure 16. Undrained triaxial compression of loose FRS 
Principal stress rotation as cause of

cyclic mobility

Jefferies, Shuttle and Been
11. $e_{\mathrm{c}}=0.974-0.0027\left(\bar{\sigma}_{\mathrm{m}}\right)^{0.614}$

Drained strength (soil properties $M_{\mathrm{tc},}, \chi_{v} \chi_{\mathrm{tc}}$ )

The strength and corresponding stress-dilatancy of FRS were determined by processing the results of the drained triaxial compression tests on the dense samples. The maximum stress ratio $\eta_{\max }$ measured is plotted against peak dilation (which occurs concurrently with peak strength and is actually $D_{\min }$ because of the 'compression positive' convention of soil mechanics) in Figure 17. A plausible trend line has been fitted through the data based on Nova's (1982) flow rule

12. $\eta_{\max }=M_{\mathrm{tc}}-(1-N) D_{\min }$

where $M_{\mathrm{tc}}$ is the critical friction ratio in triaxial compression and $N$ is a dimensionless soil property related to the work dissipation by plastic volumetric strain (Jefferies, 1997). The stress ratio $\eta$ is defined here for triaxial compression but generalises to $3 \mathrm{D}$ stress states, as does the matching dilatancy $D$ (Jefferies and Shuttle, 2002). Because the critical stress ratio $M$ varies with the intermediate principal stress, triaxial compression is taken as the reference condition to define the soil property (i.e. the constitutive model accounts for the effect of stress states other than triaxial compression). The trend line shown in Figure 17 corresponds to $M_{\mathrm{tc}}=1.45$ and $N=0.43$; there is some scatter in the test data, and correspondingly, there is a range for plausible $M_{\mathrm{tc}}, N$ combinations that might be inferred. The acceptability of the adopted combination is shown by modelling the tests and comparing the fits of the stress-strain behaviour derived from the constitutive model with that measured (shown later) - 'unthinking' regressions using the

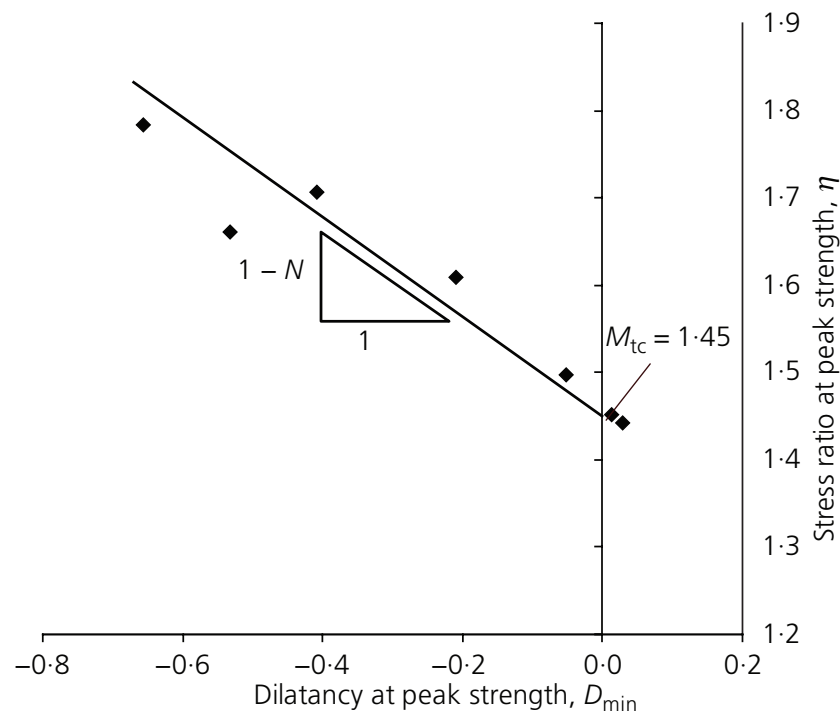

Figure 17. Stress-dilatancy of FRS at peak strength $\left(\eta_{\max }\right.$ against $\left.D_{\min }\right)$
Excel trend line function should be avoided for best assessment of soil properties with the best assessment confirmed by modelling the entire stress-strain data of the various drained tests.

As usual, the greater the dilatancy, the greater the strength. This is captured by invoking a state-dilatancy law concurrently with stressdilatancy, further generalising the framework of sand behaviour proposed by Been and Jefferies (1985). The state-dilatancy law takes the form

\section{3. $D_{\min }=\chi_{\mathrm{tc}} \psi$}

where $\chi_{\mathrm{tc}}$ is a dimensionless soil property (defined under triaxial compression) and $\psi$ is the state parameter now used as an evolving variable (the original proposal of Been and Jefferies was set in terms of initial rather than current values). The fit of this statedilatancy law to the FRS data is shown in Figure 18 with the trend line having the property $\chi_{\mathrm{tc}}=3 \cdot 4$; the trend line is forced through zero as that is a theoretical requirement (apparent offsets indicate less than optimal idealisation of the CSL; here, the power-law CSL discussed above is used).

The combination of Equations 12 and 13 define the soil strength for any combination of void ratio and confining stress, evolving as these conditions change. The proposition of Been and Jefferies (1985) amounted to a constant $\chi_{\mathrm{tc}}=3 \cdot 5$ for all sands and silty sands; in reality, this gave up some accuracy as, while true for quartz sands, soil particle mineralogy also affects the behaviour as well as gradation - thus, today triaxial testing is used to determine the state-dilatancy property $\chi_{\text {tc }}$ rather than assuming an average value.

\section{Plastic modulus (soil property $H$ )}

To fully specify the behaviour of any soil, a measure of plastic shear hardening is needed in addition to the soil properties just discussed.

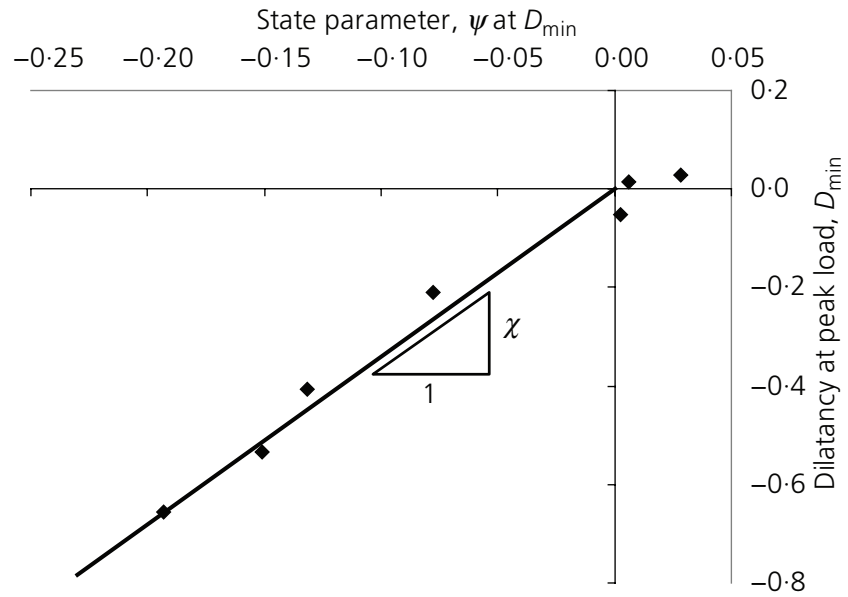

Figure 18. State-dilatancy of FRS at peak load $\left(D_{\text {min }}\right)$ (note: theory requires trend to pass through origin) 
There is presently no standard 'property' to represent plastic shear hardening (i.e. something analogous to the widely used soil normal compression index $C_{\mathrm{c}}$ ) with various soil models defining their own properties for this aspect of soil behaviour. The plastic hardening parameter $H$ - which is a dimensionless modulus - used here is determined by iterative forward modelling of entire drained triaxial tests (in a spreadsheet). In this process, an initial value of $H$ is guessed and the theoretical stress-strain behaviour is computed using NorSand, with the other soil properties just discussed remain fixed. Visual comparison of the computed and measured data leads to an improved guess for $H$, the process being iterated to best align computed and measured behaviour. A trend is generally found of the form

14. $H=H_{0}-H_{\mathrm{y}} \psi$

The $H$ relation in Equation 14 provides a good fit across the suite of FRS tests with the properties $H_{0}=60$ and $H_{\mathrm{y}}=280$. Two example calibrations on completion of this iterative procedure are shown in Figure 19 for a loose and a dense sample of FRS. Good fits are evident, fits that also confirm the adequacy of the assessed strength properties $M_{\mathrm{tc}}, N, \chi_{\mathrm{tc}}$. The NorSand simulation that is included as part of Figure 16 illustrates a comparable fit to the undrained behaviour.

\section{Summary of FRS properties}

The properties of FRS derived from the drained and undrained triaxial tests are summarised in Table 2. It is emphasised that most of these properties are not related to any particular constitutive model, although of course they do use the concept of the critical state in addition to stress-dilatancy.

Broadly, comparing these properties to other quartz sands, FRS has about a $15 \%$ greater critical friction ratio than common experience for quartz, and this is accompanied by a compressibility some three to four times larger, and with a greater volumetric coupling coefficient. In terms of micromechanics, this behaviour set is consistent with rough asperities on the soil particles, but with softer mineralogy than pure quartz.

\section{CSS tests on FRS}

\section{Methodology}

The 'undrained' (constant volume) CSS tests on samples of FRS, described in detail by Sriskandakumar (2004), were undertaken as part of the 'Earthquake induced damage mitigation from soil liquefaction' initiative (a publicly funded, multi-institutional Canadian study). The simple shear apparatus used was of the NGI type with cylindrical samples $70 \mathrm{~mm}$ in diameter and about $20 \mathrm{~mm}$ in height. Testing used stress control at applied strain rates of $10 \%$ or $20 \%$ strain per hour.

Two nominal relative densities were tested: about $40 \%$ and about $80 \%$. All samples were air-pluviated to about $40 \%$ relative density. The denser samples were then manually tamped prior to confinement being applied. The as-tested void ratio appears to not have been measured, with the work relying on carefully controlled sand
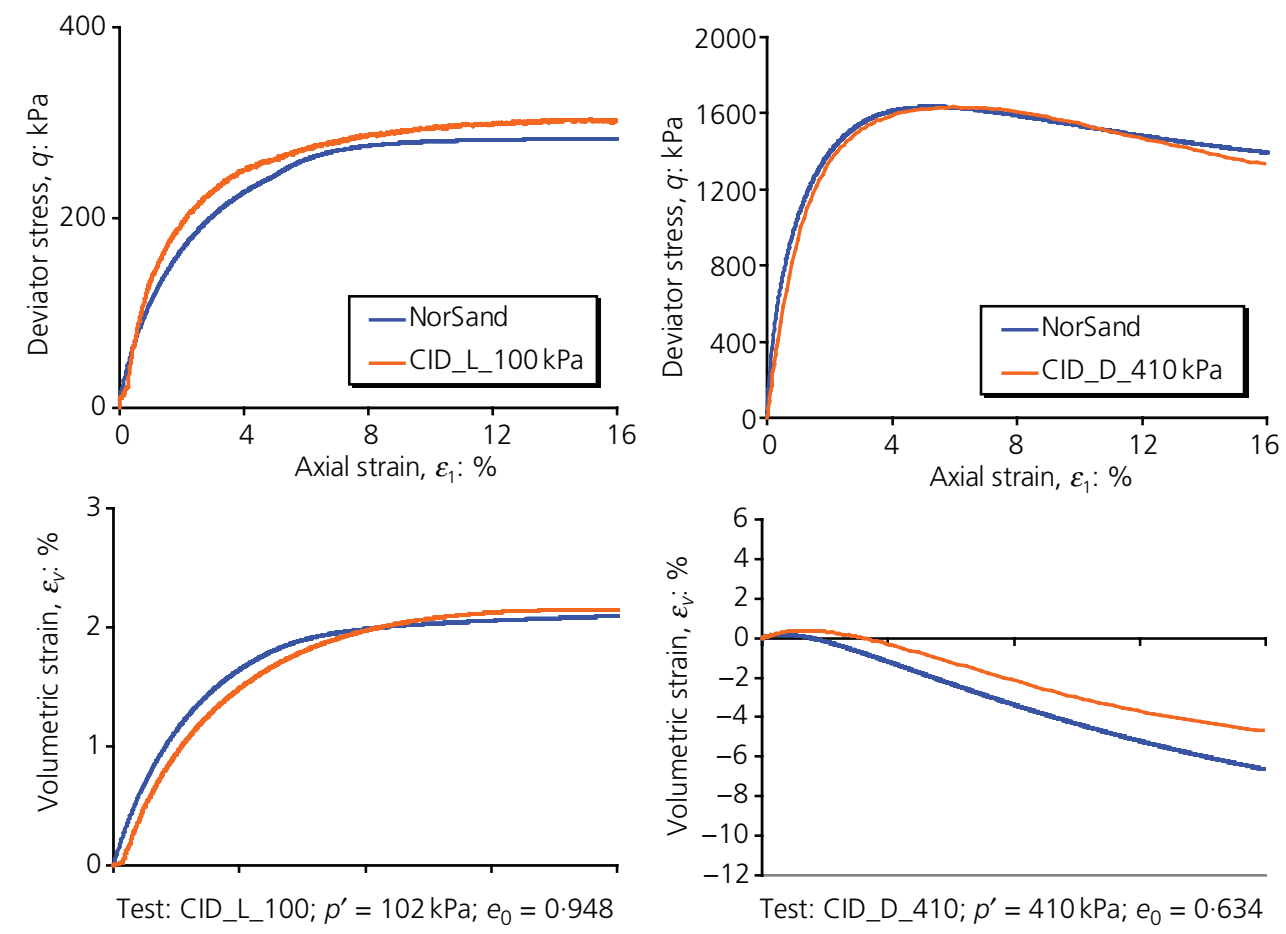

Figure 19. Example calibrations of NorSand for FRS 
placement always producing the same density in the specimen to be tested. However, the consequent accuracy in knowledge of as-tested void ratio stemming from this approach was investigated, with Sriskandakumar reporting a bandwidth of as-placed relative densities that was about five percentage points of relative density either side of the target value in a series of placement-procedure calibration tests.

The samples were consolidated to three effective vertical stress levels before shearing: 50, 100 and $200 \mathrm{kPa}$.

Digital data logging was used during testing with about 100 scans per loading cycle for each of the following: applied shear stress, shear displacement and vertical effective stress.

\section{Test program}

There are 27 CSS tests available as digital records, plus a further single monotonic simple shear test. All were constant volume ('undrained'), with four of the cyclic tests failing in the first quarter cycle (essentially making them monotonic tests). There are no duplicate tests in the available digital records. Figure 20 shows the initial conditions in these various cyclic tests on a state diagram, together with the CSL for this sand. Despite the term 'loose', all these cyclic tests were substantially dense of the critical state and the 'dense' tests were very dense. A quick inspection of the state diagram indicates none of these tests would have been prone to static liquefaction induced by shear, and indeed, that is what is found in the data.

The range of cyclic stress imposed is illustrated in Figure 21, where the CSR is compared to the initial state parameter prior to shear. This state parameter has been estimated assuming $K_{0}=0 \cdot 8$, an average of the range subsequently used in simulations when fitting NorSand to the test data and discussed shortly. This figure also

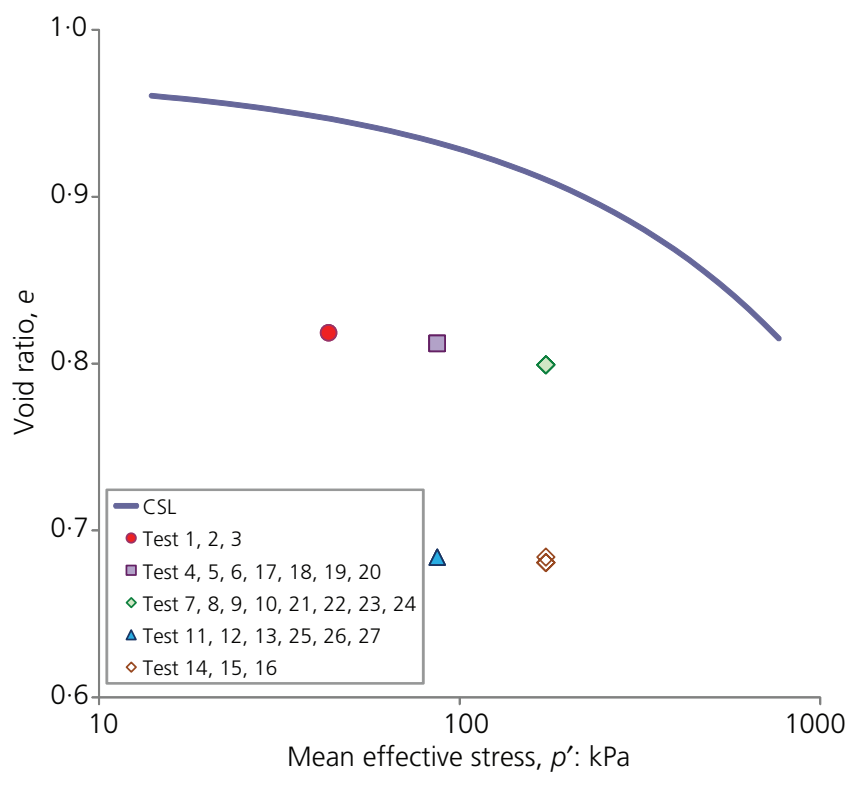

Figure 20. State diagram for CSS tests on FRS

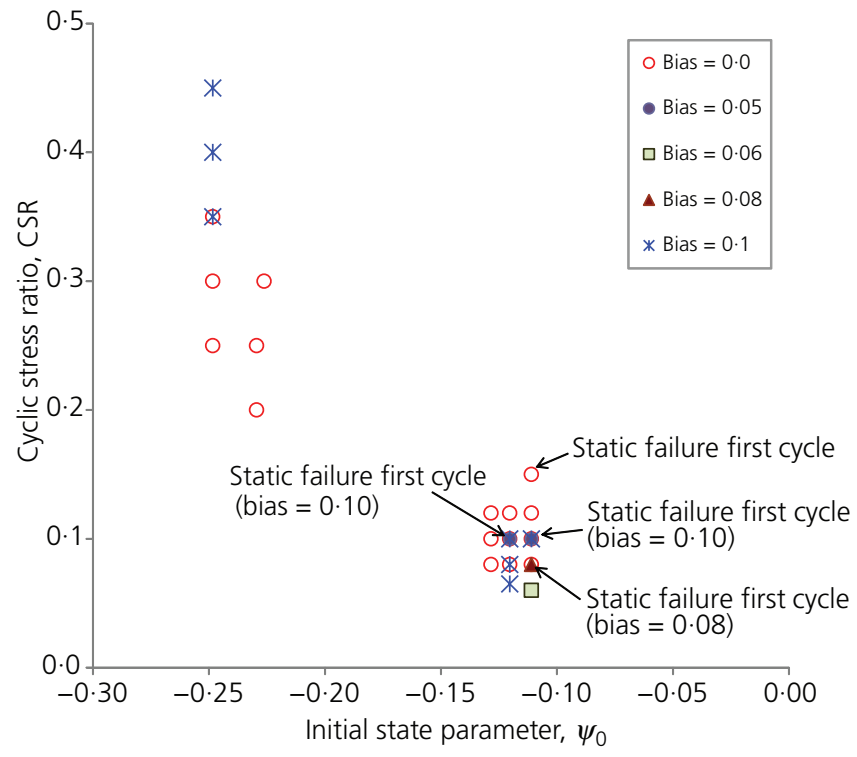

Figure 21. Summary of cyclic loading conditions on FRS

indicates the static bias used and highlights which samples failed statically in the first quarter cycle (caused by the combination of SSR and CSR producing peak shear stress exceeding the soil's undrained strength).

Test conditions are summarised in Table 3 and include the file name of the original digital data. These data files are provided as part of the downloadable spreadsheet, with a routine to view plots of the measured soil behaviour rapidly by selecting the test number.

\section{Measured soil behaviour}

A wide range of soil response in cyclic shearing was measured, ranging from 'liquefaction' in a few cycles through to what amounts to high-cycle fatigue with progressive softening but not failure itself. Examples of these two extremes of the measured behaviour are presented in Figure 22.

Although Table 3 provides the number of cycles to 'initial liquefaction', this standard condition for summarising CSS data is generally that of limiting cyclic shear strain $N_{\mathrm{L}} \Leftrightarrow \gamma_{\mathrm{xy}}>3.75 \%$ rather than $r_{\mathrm{u}}=1$. This standard condition also misleads as, in the case of the high-cycle test shown in Figure 22, there is no 'failure' as such what develops is progressive decrease in the secant shear stiffness of the soil, and even after more than 30 cycles that secant stiffness may be satisfactory: it is a 'serviceability state', not an 'ultimate state'.

\section{Simulation of CSS}

\section{Validation for monotonic simple shear}

NorSand has seen extensive validation, drained and undrained, for fixed principal stress direction, both for various triaxial paths (Jefferies and Shuttle, 2005) and plane strain (Jefferies and Shuttle, 


\begin{tabular}{|c|c|c|c|c|c|c|c|}
\hline No. & Data file & $\bar{\sigma}_{v 0}: \mathrm{kPa}$ & CSR & SSR & $e_{0}^{c}$ & $\psi_{0}^{\mathrm{a}}$ & $N_{L}$ \\
\hline 1 & DSS38-50-0.08 & 50 & 0.08 & 0.00 & $0 \cdot 818$ & $-0 \cdot 128$ & $12 \cdot 0$ \\
\hline 2 & DSS38-50-0.1 & 50 & $0 \cdot 10$ & 0.00 & $0 \cdot 818$ & $-0 \cdot 128$ & $2 \cdot 5$ \\
\hline 3 & DSS38-50-0.12 & 50 & $0 \cdot 12$ & 0.00 & $0 \cdot 818$ & $-0 \cdot 128$ & $1 \cdot 6$ \\
\hline 4 & DSS40-100-0.08 & 100 & 0.08 & 0.00 & $0 \cdot 812$ & $-0 \cdot 120$ & $17 \cdot 1$ \\
\hline 5 & DSS40-100-0.1 & 100 & $0 \cdot 10$ & 0.00 & $0 \cdot 812$ & $-0 \cdot 120$ & $6 \cdot 2$ \\
\hline 6 & DSS40-100-0.12 & 100 & 0.12 & 0.00 & $0 \cdot 812$ & $-0 \cdot 120$ & $3 \cdot 1$ \\
\hline 7 & DSS44-200-0.08 & 200 & 0.08 & 0.00 & 0.799 & $-0 \cdot 111$ & $33 \cdot 6$ \\
\hline 8 & DSS44-200-0.1 & 200 & $0 \cdot 10$ & 0.00 & 0.799 & $-0 \cdot 111$ & $7 \cdot 2$ \\
\hline 9 & DSS44-200-0.12 & 200 & 0.12 & 0.00 & 0.799 & $-0 \cdot 111$ & $3 \cdot 6$ \\
\hline 10 & DSS44-200-0.15 & 200 & 0.15 & 0.00 & 0.799 & $-0 \cdot 111$ & $0.25^{b}$ \\
\hline 11 & DSS80-100-0.25 & 100 & 0.25 & 0.00 & 0.684 & $-0 \cdot 248$ & $46 \cdot 0$ \\
\hline 12 & DSS80-100-0.30 & 100 & $0 \cdot 30$ & 0.00 & 0.684 & $-0 \cdot 248$ & $19 \cdot 7$ \\
\hline 13 & DSS80-100-0.35 & 100 & 0.35 & 0.00 & 0.684 & $-0 \cdot 248$ & $7 \cdot 7$ \\
\hline 14 & DSS81-200-0.2 & 200 & 0.20 & 0.00 & 0.681 & $-0 \cdot 229$ & $90 \cdot 0$ \\
\hline 15 & DSS81-200-0.25 & 200 & 0.25 & 0.00 & 0.681 & -0.229 & $20 \cdot 0$ \\
\hline 16 & DSS80-200-0·3 & 200 & $0 \cdot 30$ & 0.00 & 0.684 & -0.226 & $5 \cdot 7$ \\
\hline 17 & DSS40-st0.1-100-0.08 & 100 & 0.08 & $0 \cdot 10$ & $0 \cdot 812$ & $-0 \cdot 120$ & $3 \cdot 2$ \\
\hline 18 & DSS40-st0.1-100-0.065 & 100 & 0.07 & $0 \cdot 10$ & $0 \cdot 812$ & $-0 \cdot 120$ & $15 \cdot 2$ \\
\hline 19 & DSS40-st0.05-100-0.1 & 100 & $0 \cdot 10$ & 0.05 & $0 \cdot 812$ & $-0 \cdot 120$ & $2 \cdot 7$ \\
\hline 20 & DSS40-st0.1-100-0·1 & 100 & $0 \cdot 10$ & $0 \cdot 10$ & 0.812 & $-0 \cdot 120$ & $0.25^{b}$ \\
\hline 21 & DSS44_st0p05_200_0p1 & 200 & $0 \cdot 10$ & 0.05 & 0.799 & $-0 \cdot 111$ & $5 \cdot 3$ \\
\hline 22 & DSS44_st0p1_200_0p06 & 200 & 0.06 & 0.06 & 0.799 & $-0 \cdot 111$ & $57 \cdot 1$ \\
\hline 23 & DSS44_st0p1_200_0p08 & 200 & 0.08 & 0.08 & 0.799 & $-0 \cdot 111$ & $0.25^{b}$ \\
\hline 24 & DSS44-st0 $1-200-0 \cdot 1$ & 200 & $0 \cdot 10$ & $0 \cdot 10$ & 0.799 & $-0 \cdot 111$ & $0.25^{b}$ \\
\hline 25 & DSS80-st0.1-100-0.35 & 100 & 0.35 & $0 \cdot 10$ & 0.684 & $-0 \cdot 248$ & $31 \cdot 5$ \\
\hline 26 & DSS80-st0.1-100-0.40 & 100 & 0.40 & $0 \cdot 10$ & 0.684 & $-0 \cdot 248$ & $18 \cdot 2$ \\
\hline 27 & DSS80-st0.1-100-0.45 & 100 & 0.45 & $0 \cdot 10$ & 0.684 & $-0 \cdot 248$ & $8 \cdot 2$ \\
\hline
\end{tabular}

a Computed assuming $K_{0}=0.8$

${ }^{b}$ Failed on first loading

${ }^{c}$ Computed using $e_{\min }=0.62$ and $e_{\max }=0.94$

Table 3. CSS tests of Fraser River sand

2002; Wanatowski et al., 2013). However, the simple shear apparatus is quite different from the equipment used for these validations and further validation under monotonic simple shear is appropriate, indeed even necessary, before moving to the more complex situation of CSS. There is one such test available in the digital records, an undrained monotonically loaded test at an initial vertical effective stress of $100 \mathrm{kPa}$ on a sample at nominally $40 \%$ relative density (corresponding to an initial state parameter, $\psi_{0} \approx-0 \cdot 1$ ).

The initial horizontal stress is not known. If a first estimate is taken as a common expectation that $K_{0} \approx 1-\sin (\phi)$, then a starting point could be $K_{0}=0 \cdot 5$. The result of using this value, with the other properties derived from triaxial calibration (Table 2) and the inferred void ratio from the reported relative density, is shown in Figure 23. The computed stress path in $\tau_{\mathrm{xy}}$ against $\sigma_{\mathrm{y}}^{\prime}$ space is a poor match. A reason for the poor match is that $K_{0}=0.5$ is associated with substantial initial deviatoric stress (applied drained) before the undrained shearing commences, as illustrated in Figure 24. The effect of undrained conditions is therefore less than perhaps what might be expected from consideration of isotropic triaxial tests. Decreasing the initial deviator stress, which is the same thing as increasing $K_{0}$, improves the simulation results with Figures 23 and 24 both showing the effect of the change to $K_{0}=0.9$; an improved fit to the stress path is evident but there remains too much dilation.

The ethos of critical state models is that the soil's measured void ratio must be honoured. But, there is an issue over 'measured' in the case of the simple shear tests on FRS, as the reported void ratios (which must be inferred from the reported relative density, $e_{\min }$ and $e_{\max }$ ) were never measured for any particular test. Instead, the achieved relative density was calibrated to the sample preparation method (sand drop height and flow rate) with that calibrated preparation method then being relied on. There is a further issue over the CSL itself, discussed in the 'Critical state locus' section, where plane strain may affect the CSL derived under triaxial conditions - equivalent to a 

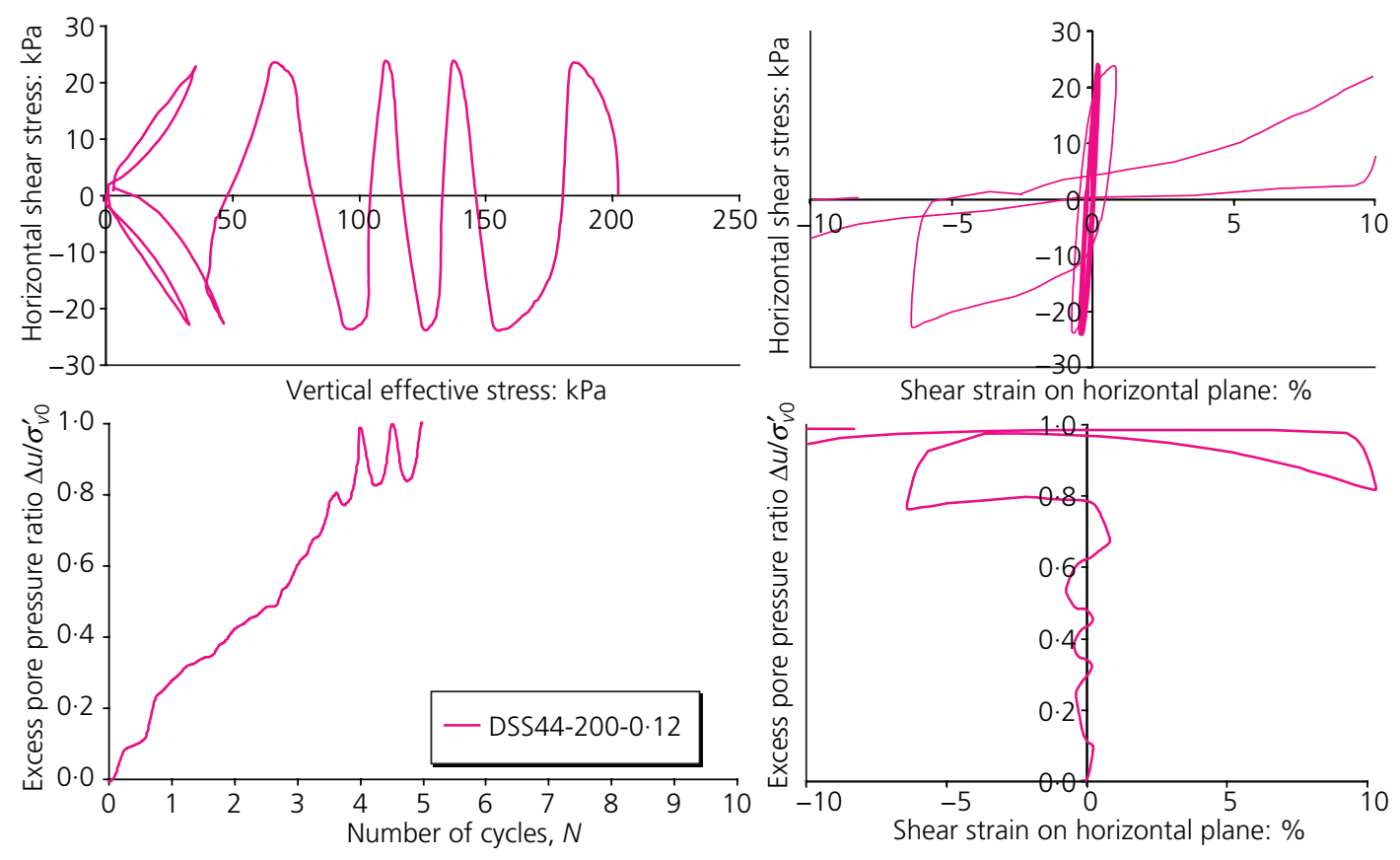

(a)
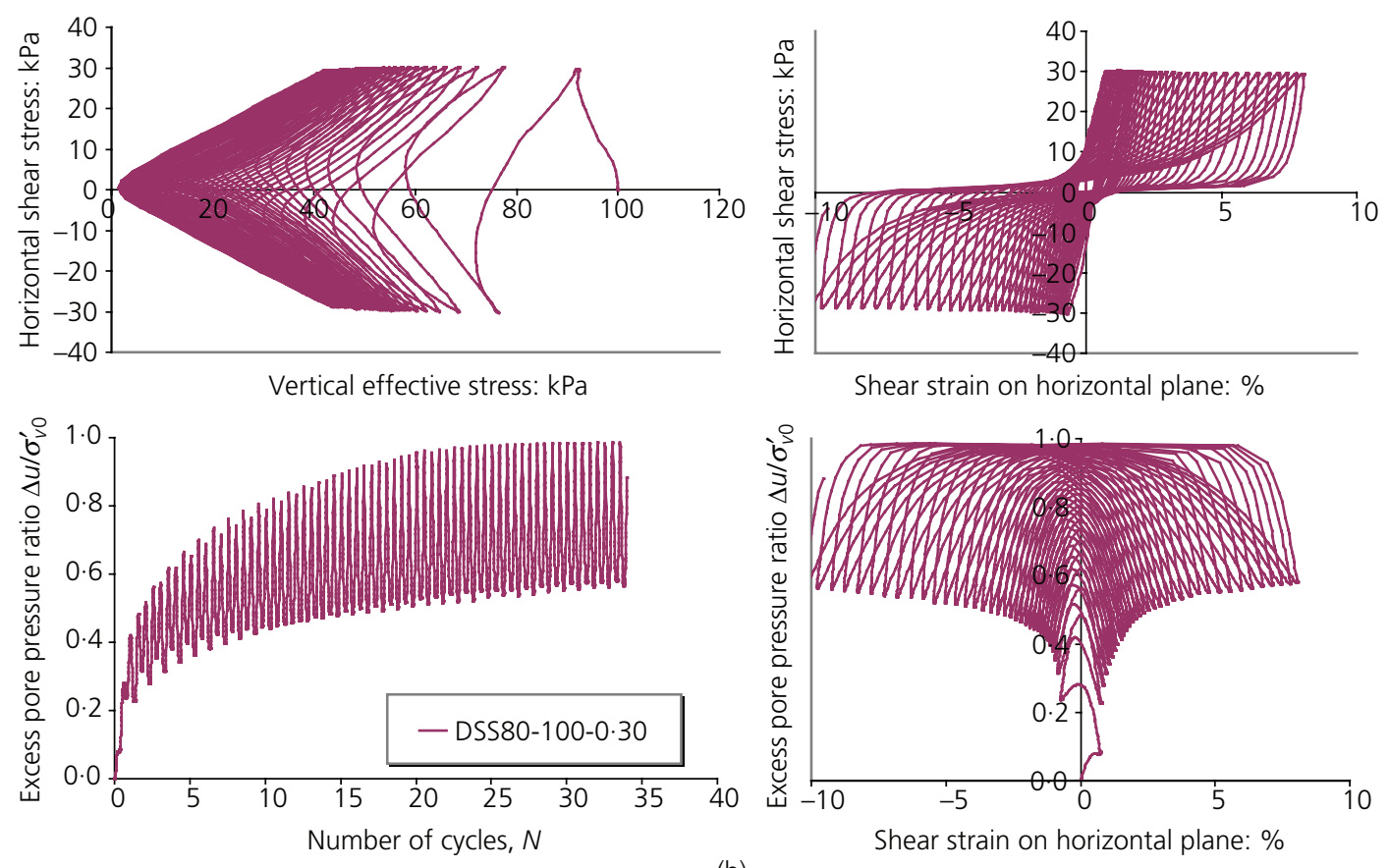

(b)

Figure 22. Illustration of range of measured FRS behaviour in CSS; (a) test with shear failure after four loading cycles; (b) test with high-cycle fatigue-like response

shift in the soil property $\Gamma$, by about 0.05 , in changing from triaxial $\left(\theta=30^{\circ}\right)$ to plane strain $\left(\theta \sim 15^{\circ}\right)$. Both uncertain $e_{0}$ and uncertain $\Gamma$ can be investigated by loosening the constraint on $\psi_{0}$, as that is their net effect; Figure 25 shows the effect of changing to a looser state of $\psi_{0}=-0 \cdot 02$, which is about the credible limit of the uncertainty. The computed stress-strain behaviour is improved, but to nothing like the quality of validation found in triaxial compression (Figure 16).

There is now a question about the quality of the simple shear apparatus itself. Issues such as the effect of non-uniform strains were discussed in 

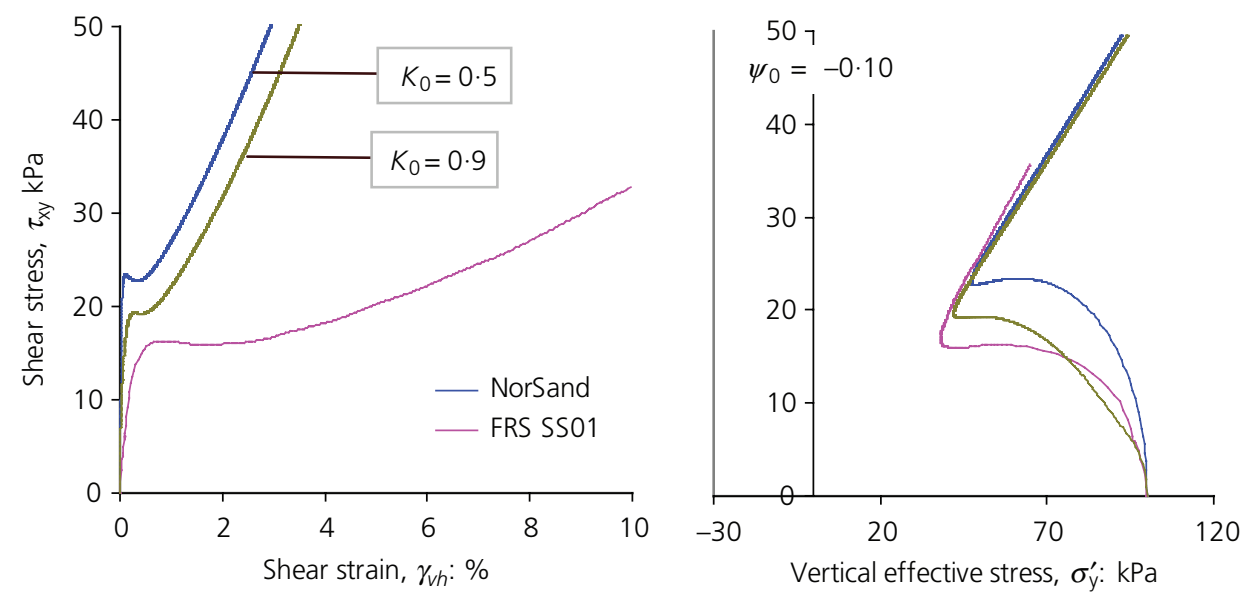

Figure 23. Example fits to monotonic undrained SS showing effect of $K_{0}$

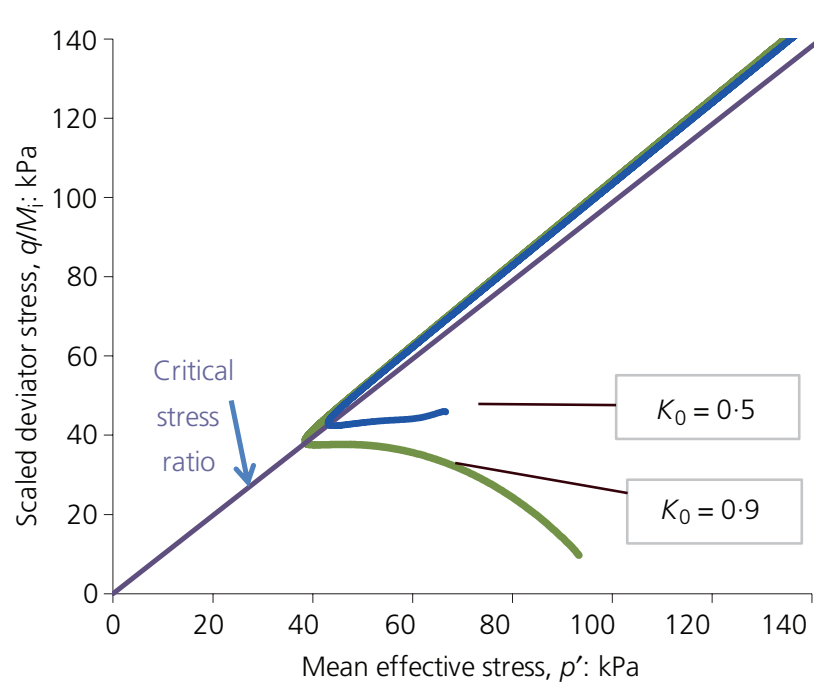

Figure 24. Effect of $K_{0}$ on initial deviatoric stress in simulation of undrained SS

the section headed 'Why PSR is important' but the particular concern now is the adequacy of the lateral restraint. The numerical simulation reported here enforces $\varepsilon_{\mathrm{x}}=0$ to a precision of one part in $10^{7}$. In contrast, the testing reported the reinforced rubber membrane as 'stiff enough to constrain any lateral deformations' without quantification. The effect of violating the 'stiff enough' constraint was mimicked by reducing both the elastic and plastic moduli by $50 \%$ (they have to be moved together else the stress path is lost), with the results shown in Figure 26. A pleasing fit is now evident.

In summary, there is uncertainty in the inferred initial state of these simple shear tests on FRS, with a net change of $\Delta \psi_{0} \sim+0.05$ being a plausible consequence. There is also a very substantive cause for concern over the adequacy of the reinforced rubber membrane to impose sufficient confinement for sands (recall that this test apparatus was originally devised for soft clay).

\section{Fitting methodology for CSS}

The basic premise of critical state models, including NorSand, is that soil properties do not vary with void ratio or confining stress: the calibration reported earlier and giving the properties shown in Table 2 must be used 'as is'. This restriction is relaxed by halving the elastic and plastic moduli based on the validation to the monotonic simple shear tests as just discussed. However, that leaves two 'free' parameters that may be varied to fit the NorSand model to the data: the initial geostatic stress ratio, $K_{0}$, and the PSR softening modulus, $Z$. Fitting of the NorSand model to each cyclic test is done by forward modelling, just as used to determine $H$ trends in the triaxial calibration, where the initial values of the 'free' parameters are guessed, simulations carried out and the guesses updated - the simulations continuing until a visually pleasing fit of model to data is achieved.

Of these free parameters, $K_{0}$ is an unmeasured initial condition in the tests while $Z$ is the new soil property whose values and trends are sought from the suite of these CSS tests. Practically, $K_{0}$ is constrained by the maximum excess pore pressure, as the end-condition of the cyclic model is a strong function of $K_{0}-$ it is not difficult to establish values consistent with the measured soil behaviour in each test. The soil property $Z$ controls the rate at which excess pore pressure develops per loading cycle and is a well-constrained property.

\section{Simulation results}

The NorSand simulation of CSS shown earlier in Figure 10 is actually a calibration to the FRS data (it is for test number 5 in Table 3 ). This was computed using $Z=10 \cdot 2$ and $K_{0}=0 \cdot 9$; smaller values of $K_{0}$ were unable to reproduce anything near the final excess pore pressure. 


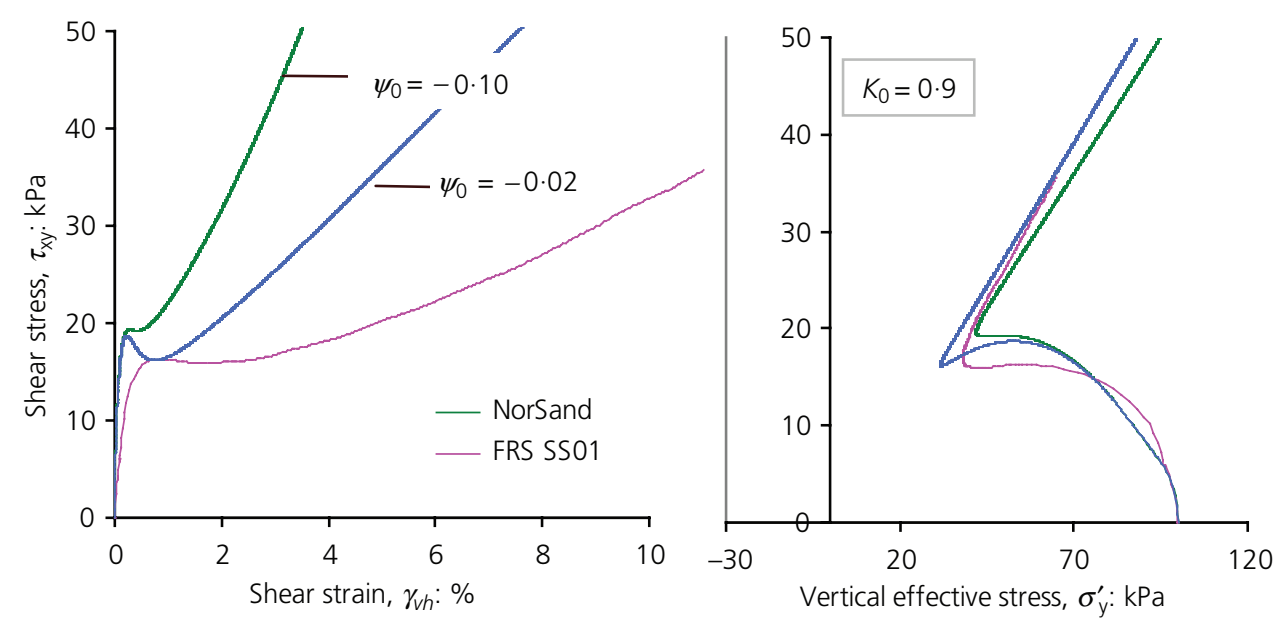

Figure 25. Effect of initial state on simulation of undrained SS
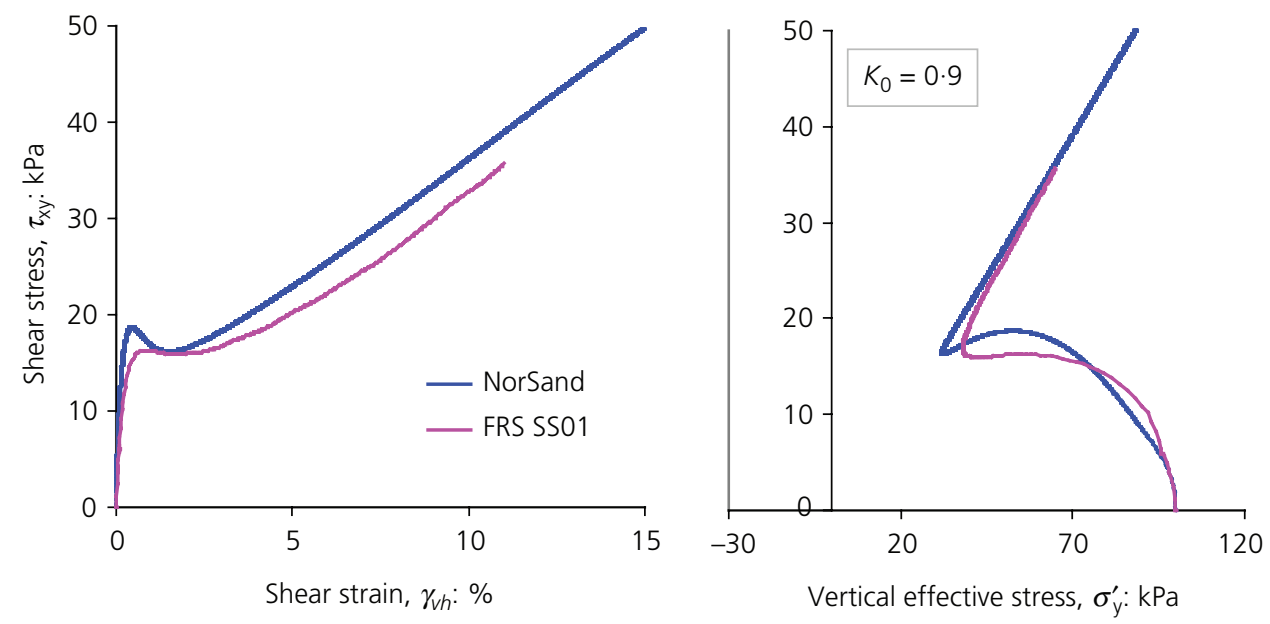

Figure 26. Effect of reduced lateral restraint on fit to undrained SS test

One of the major issues in the conventional assessment of soil liquefaction is the effect of static bias, or ' $K$ alpha', as it is commonly called after the factor of that name in the NCEER assessment method (Youd et al., 2001). A reasonable first question is then: how well does annealing by PSR capture the effect of static bias on soil behaviour? Figure 27 shows the answer, this being test number 21 of the FRS data, which had static bias such that the shear stress reversed its sense with each load cycle. With change to $Z=5$ and using that tests $e_{0}$ and $\sigma_{\mathrm{y} 0}^{\prime}$, the fit shown in Figure 27 was obtained. The rate of excess pore pressure development is nicely captured, as is the transition into substantial plastic strains. But, just as seen earlier, the PSR-annealing algorithm leads to a steady cyclic state that does not replicate the very high $r_{\mathrm{u}}$ exhibited by the soil at the late stage of the test.

Moving to progressively more static bias, Figure 28 shows a test where the static bias is so large that the applied shear stress never reverses its sense. Achieving a fit to the increase of excess pore pressure with load cycling needs about a doubling of $Z$ from the basic no-bias situation. This is a further example of the constitutive model unloading elastically because the deviator stress $q$ remains within the yield surface (there is no change to the mean stress during elastic loading and unloading).

Now turning to one of the very dense tests (test number 16), Figure 29 compares the simulation using $Z=5$ to the data. There are several aspects of concern, both with the fit of the model to the data and in the data itself. Dealing with the data first, inspection of the initial loading cycle shows the sand to be dilating - but this must be at a very low stress ratio since the true critical stress ratio that demarks the transition to dilation is well-established later in the test by the measured 'butterfly' loops in the stress path. The second discrepancy, also commented on in the other tests, is that the tested sand yields in elastic unloading whereas the model does not. The model accumulates plastic strain within a fairly stiff response for each cycle, in contrast to the onset of extreme plasticity in the actual sand response at what is 

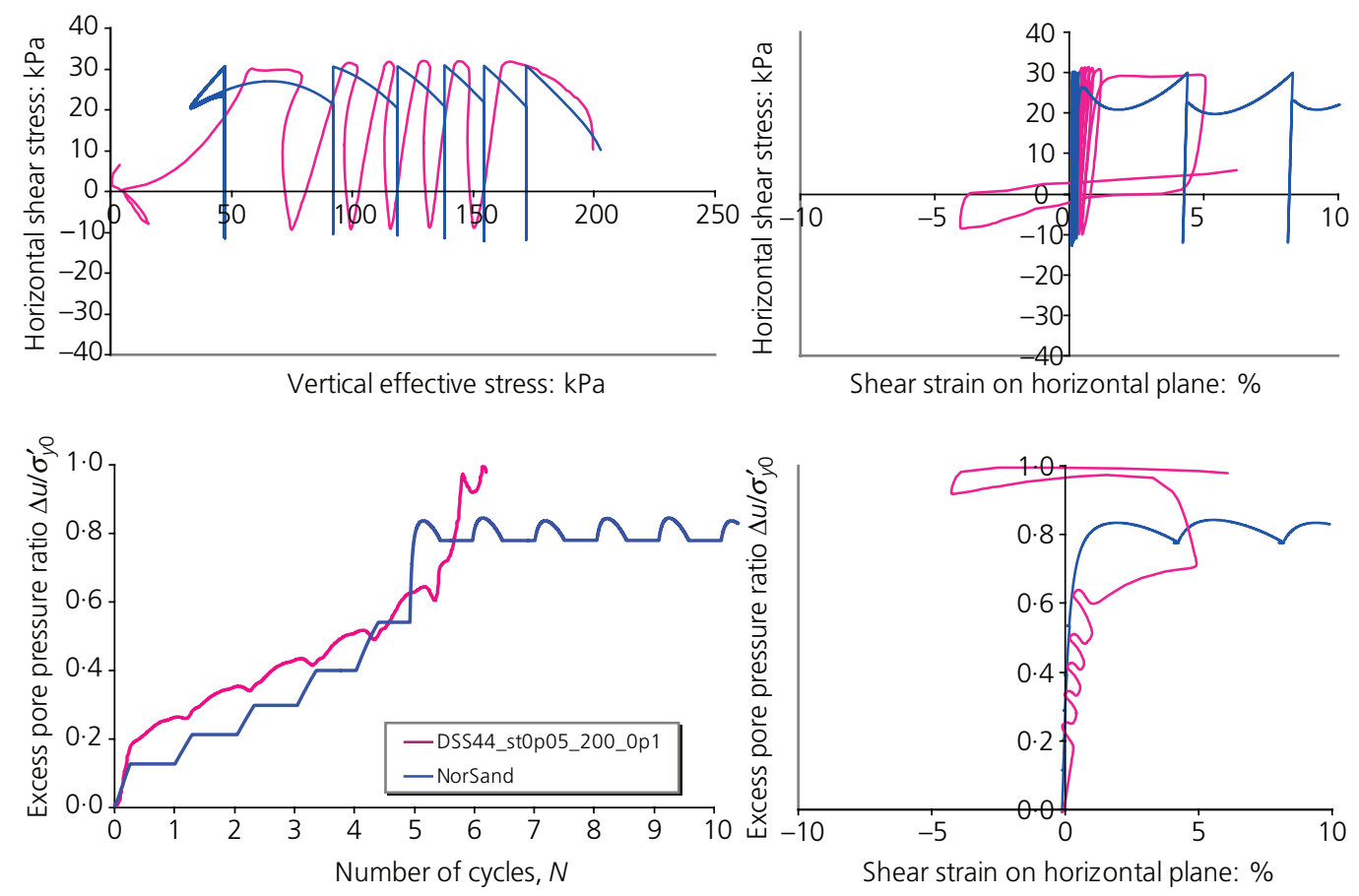

Figure 27. Simulation for CSS test with moderate static bias
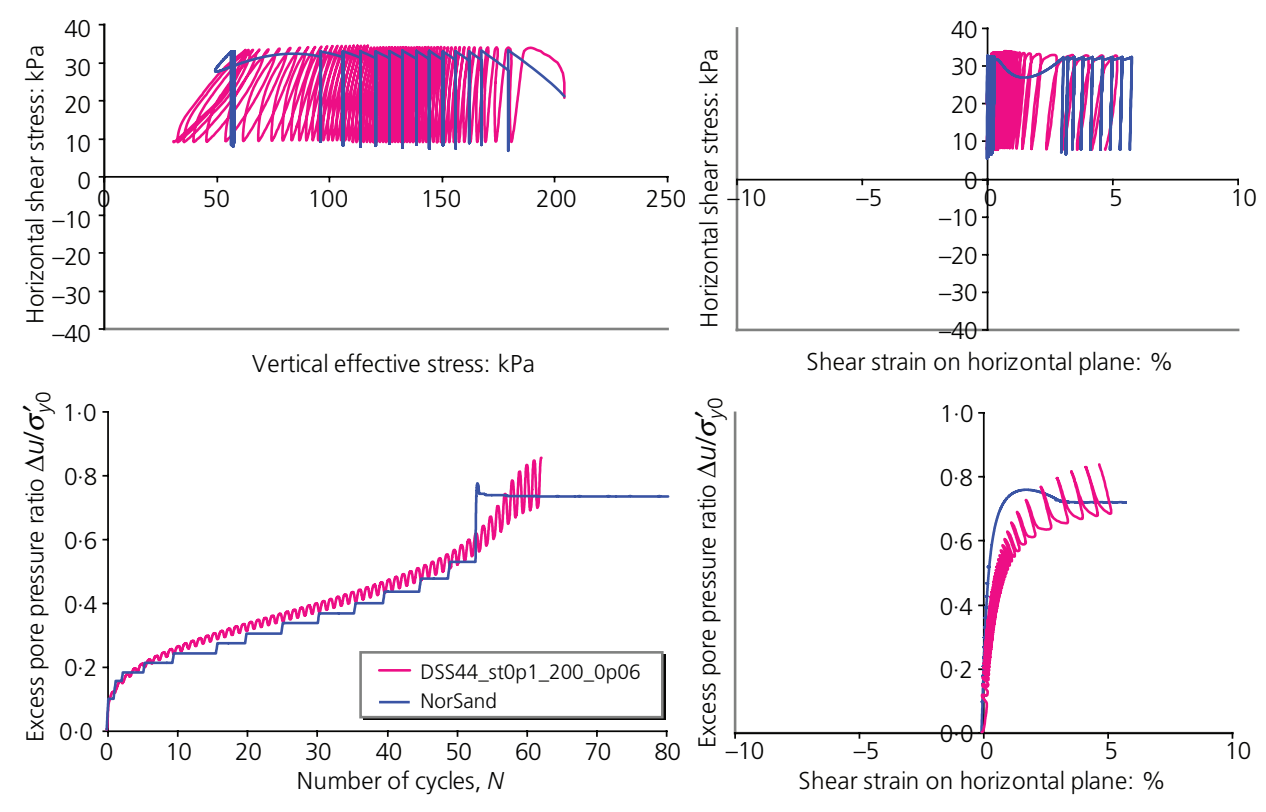

Figure 28. Simulation for CSS test with large static bias

essentially a minimum deviator stress for that load cycle. Theoretical implications of this mismatch are discussed below.

\section{Effect of $K_{0}$}

The geostatic stress ratio needed for the simulations to capture measured data was universally far larger than what would have been anticipated from Jaky's formula. Broadly, $K_{0} \sim 0.9$ fit the loose tests with $K_{0} \sim 0.7$ fitting the denser tests (depending on to what extent it is preferred to fit the final pore pressure rather that the rate of increase in pore pressure). There is little ambiguity in these estimates, as is readily verified by downloading the spreadsheet and trying lesser values - as the $K_{0}$ is lowered, so is the peak excess pore pressure ratio $u_{\mathrm{e}} / \sigma_{\mathrm{y} 0}^{\prime}$. 

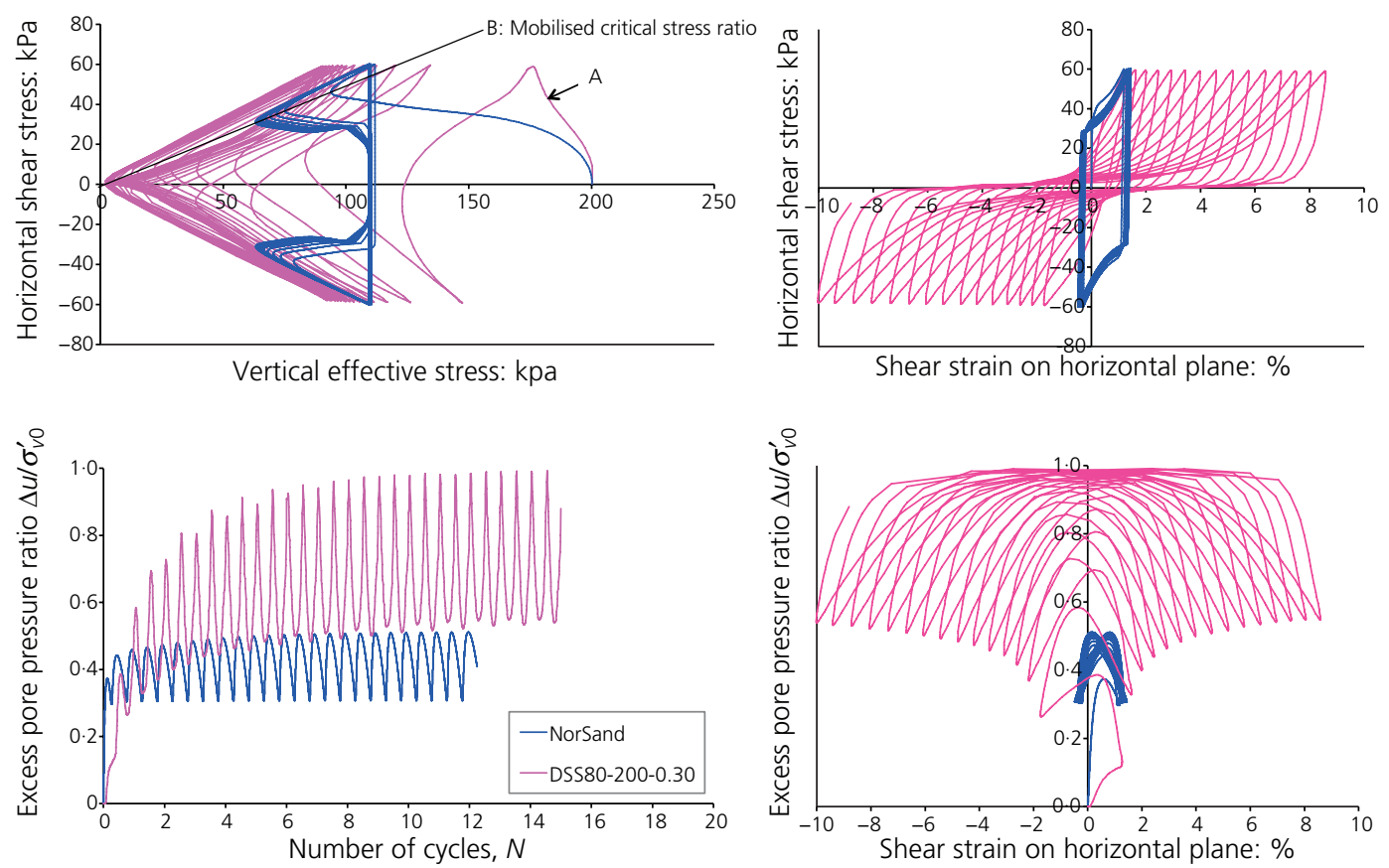

Figure 29. Simulation for CSS test on very dense FRS

It is also worth noting that there are limitations with the accuracy of the CSS test equipment when substantive stiffness degradation develops (associated with the late-stage 'butterfly' stress paths). The condition $u_{\mathrm{e}} / \sigma_{\mathrm{y} 0}^{\prime} \sim 1$ implies tension in $\sigma_{\mathrm{x}}^{\prime}-$ an absurd situation for particulate materials. The best-estimate of $K_{0}$ depends on the extent to which the measured data is taken as 'ground truth'; increasing concern about the late-stage accuracy of the CSS data leads to reduced numerical values for the bestestimate for $K_{0}$.

\section{Trends in Z}

There is a systematic difference in the softening modulus between the loose and dense tests.

In the case of the loose tests, Figure 30 provides a map of the best-fit values for $Z$ where the fit was based on optimising the rate of increase in excess pore pressure with number of cycles (' $N$ '). The estimates for $Z$ in each loose test are plotted against the peak stress ratio $(=\mathrm{CSR}+\mathrm{SSR})$ imposed. A linear trend is

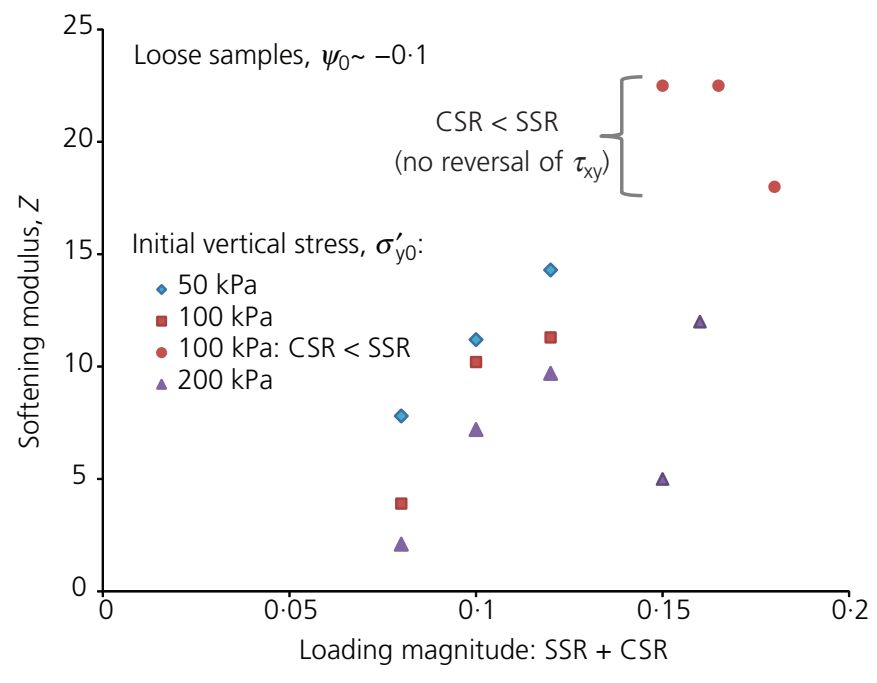

Figure 30. Trends for softening modulus 
evident, spanning the cyclic tests and those with sufficient static bias such that the imposed shear stress never reversed direction (highlighted in the figure). There is a slight bias with initial vertical effective stress, with parallel trends being evident for different stress levels.

In the case of the dense tests, $Z \sim 6$ fit all the simulations, but that fit is misleading. As illustrated earlier, these dense tests in the FRS database had rather extreme cyclic stress ratios imposed and transitioned into being dilation-dominated within one to two loading cycles. Most of the data for these dense tests are not about how excess pore pressure develops as the samples are brought to shear failure, but rather what happens in unloading after intense shear. Equation 7 does not, and cannot, address that issue as the postpeak stress path is reducing $\bar{\sigma}_{q}$ at constant $\bar{\sigma}_{\mathrm{m}}$. Correspondingly, the estimate for $Z$ is poorly constrained.

\section{Discussion}

The proposition explored in the present work is that PSR softens (anneals) the yield surface of a soil. This proposition follows from the original contribution of Arthur and co-workers, being a mathematical characterisation of their seminal insight. It can also be remarked that annealing is a common aspect of metals, often relied on during fabrication of components - it is just unusual in the context of soils.

A simple expression of this idea, which is no more than an additional term to the hardening law in a general critical state soil model, captures much of the behaviour of soil in CSS for lightly dilatant samples, and using soil properties determined from standard calibration to triaxial compression tests. However, the simple representation of annealing implemented does not capture everything, and aspects that are not captured provide insight on soil behaviour - now discussed.

\section{Dilation at low $\eta$ during loading}

The dense cyclic tests, of which Figure 29 shows an example of the common behaviour, dilate at small values of $\tau_{\mathrm{xy}}$ almost regardless of the vertical effective stress $\sigma_{y}^{\prime}$ (e.g., at point 'A' in Figure 29). Conversely, the final stages of the measured 'butterfly' loops on the stress path are close to the transition stress ratio $\eta=M$ which, under current understanding of stress dilatancy, would be the condition separating contractive from dilative strains. The contraction/ dilation condition is intrinsic to the NorSand simulations, and those simulations confirm the location of the transition stress ratio inferred from inspection of the data (the line annotated as ' $\mathrm{B}$ ' in Figure 29). How is it then possible for dilation, so very pervasive in the measured stress paths of the dense samples, to develop at so much smaller ratios of $\tau_{\mathrm{xy}} / \sigma_{\mathrm{y}}^{\prime}$ ?

One possibility, and which also addresses concerns over the inferred magnitude of $K_{0}$, is that these samples have initially a very low value of $K_{0}$, which implies substantial mobilised $\eta$ as illustrated in Figure 24. Then, as the yield surface anneals, there is not just a reduction in its size to reflect the new loading direction but PSR in itself causes proportionally more horizontal plastic strains than vertical. In effect, this leads to an evolving $K_{0}$ with number of load cycles.

\section{Yield in unloading}

Again, looking at the dense test shown in Figure 29, consider the result of reducing shear stress after the first quarter cycle (i.e. the first unloading point). Notice that the measured vertical effective stress (magenta line) decreases in this 'unloading' and indeed this reduction in $\sigma_{\mathrm{y}}^{\prime}$, equivalent to excess pore pressure, is more than what occurred in the initial loading. There is substantial yield in unloading. This unloading yield is present - to a greater or lesser extent - in every test within the FRS. Other published CSS tests on other soils also have this feature, and it would seem unlikely that there it is an artefact of the particular test apparatus used for the tests discussed here.

Now consider the elastic stress path during reversal of load direction. Reducing $\tau_{\mathrm{xy}}$ induces reducing $\bar{\sigma}_{q}$ and this develops at constant $\bar{\sigma}_{\mathrm{m}}$ (because of elasticity). How can yielding develop for such a stress perturbation?

NorSand does yield in unloading, and with considerable detail in terms of triaxial tests (Jefferies, 1997), but only when stress paths encounter the inner cap (see Figure 9). The elastic stress changes in unloading during CSS do not intersect NorSand's inner cap, so the inner cap does not provide a mechanism for the observed yielding.

It might be argued that what is required is to introduce a lower boundary to the yield surface, say a line in stress space from the origin at a slope of $M / 2$. Adding such a feature would capture much of what is seen in a CSS test. But such an additional inner boundary to the yield surface would also conflict with the behaviour of sand in isotropic loading/unloading (e.g., see the data in the paper by Jefferies and Been (2000)), and such an inner yield condition would also throw away all the understanding of a critical state framework - still the best framework available for linking void ratio to soil behaviour - as that understanding requires a single closed yield surface that intersects the isotropic compression axis (i.e. the original proposition of Drucker et al. (1957)).

Of course, there are kinematic hardening (or 'bubble') models where the yield surfaces move location in stress space and which could also be made to respond to PSR. However, there is a reasonable view that such models are too complicated - here addition of a simple softening rule to a simple general critical state model captures much of the measured behaviour. Would it not be better to continue down this path and consider a modest change to the softening rule rather that concluding that complex kinematic hardening models are necessary?

An aspect worth considering is to modify the suggestion of Arthur and co-workers, and change to controlling the annealing of the yield surface using the strain increment direction rather than the rotation of principal stress. This strain increment direction always reverses in the CSS test, at peak shear stress, even for tests with 
such static bias that the principal stress direction does not change from $\alpha=45^{\circ}$ after the first few cycles. As such, using changes in the strain increment direction to soften the yield surface offers the possibility of capturing those aspects of soil behaviour that are missed by adopting an annealing rule entirely in terms of changes in principal stress direction.

One way forward would be to soften the yield surface using the angular difference between current major principal stress direction and that of the major principal strain increment direction. Making the recognised non-coaxiality of strain increment and stress (which both change direction) the additional 'loading measure' for the yield surface (rather than PSR on its own) will retain what already works well, while responding slightly differently to the insights raised by Arthur and co-workers.

\section{Adequacy of Equation 7}

Equation 7 was proposed as a simple softening rule consistent with critical state ideas. However, as discussed in the 'Critical state locus' section, there are other views and in particular the possibility of other state measures influencing the CSL.

A proper softening rule should automatically deal with the effect of differing CSR and SSR imposed in the CSS test. Figure 30 indicates that the proposed rule partially does not do this, as the softening modulus is not constant but correlates with the imposed cyclic stress conditions. Equation 7 does include the mobilised stress conditions by means of the image stress ratio, but Figure 30 suggests that perhaps a further multiplier of $\eta / M_{\mathrm{i}}$ is needed, although this is a secondary consideration to modifying the 'driver' for annealing as just discussed.

A further concern for Equation 7 is whether it should include the state parameter. State is intrinsic to cyclic loading through its control of yield surface hardening, but does not have to appear in the annealing rule despite its attractiveness from an idealised viewpoint about the nature of the critical state. However, again, the concern is that the primary issue is what drives the annealing rule and further developments are needed in this area first.

\section{Adequacy of NGI apparatus}

The reinforced rubber membrane from the CSS test has become the dominant commercial variety, with much equipment commissioned in the past decade. In fact, it is the apparatus that has become the standard of practice. But 'standard' does not mean the data from the test should be used at face value for engineered construction. First, further work is needed on the effect of membrane compliance on measured sand behaviour. Certainly, the simulations presented in this paper suggest that there is a problem with membrane compliance reducing the effect of the excess pore pressure on the soil response. Second, there is a serious issue around the effect of $K_{0}$ and the differing conditions between the test and actual conditions.

Most generally, engineers need to acknowledge that the simple shear test is supplementary to the triaxial test and that simple shear test data need modelling to understand what each test result means. The downloadable spreadsheet is a template for that, and it is easy enough to switch out NorSand for an alternative model (say a statebased bounding surface variant) if that is preferred. The important thing is to recognise that the simple shear tests are incomplete data, and only modelling that data allows proper inference of the soil behaviour being measured.

The idea that CSS data need to be modelled to infer the soil behaviour from the test data produces one final consideration. For all its defects, the current form of the CSS test is now a given for engineering practice. But researchers are not so constrained. A lot of theoretical understanding could be gained about CSS by using a different form of this equipment from the NGI type and of sufficient size to include miniature total pressure cells such as those implemented by Wanatowski and Chu (2007) in their update of Cornforth's plane strain apparatus.

\section{Conclusion}

PSR has been known to act as 'loading' on soils since the seminal work of Arthur et al. some 30 years ago. However, their key insight - that soils yield under constant stress invariants if the principal stresses rotate - has been consistently neglected in virtually all constitutive models of soil and all procedures/ methods of geotechnical engineering practice. This neglect is a pity, as a rather simple softening (annealing) of yield surface size, proportional to the rotation of principal stress, captures much of soil behaviour that is measured in the CSS test as demonstrated in this paper using the NorSand model. Equally, the implementation of PSR-annealing makes clear that there is a further loading process for soil: PSR annealing is not sufficient to capture all aspects of soil behaviour in the CSS test. It is suggested that rotation of the strain increment direction may be the missing additional factor. Hopefully, this paper will arouse interest in further developments to understand the now practically important CSS test.

\section{REFERENCES}

Airey DW and Wood DM (1987) An evaluation of direct simple shear tests on clay. Géotechnique 37(1): 25-35.

Arthur JRF and Menzies BK (1972) Inherent anisotropy in sand. Géotechnique 22(1): 115-128.

Arthur JRF, Chua KS and Dunstan T (1979) Dense sand weakened by continuous principal stress direction rotation. Géotechnique 29(1): 91-96.

Arthur JRF, Chua KS, Dunstan T and Rodriguez JI (1980) Principal stress rotation: a missing parameter. Journal of Geotechnical Engineering, ASCE 106(GT4): 419-433.

ASTM (American Society for Testing and Materials) (2000) D4254: Standard test methods for minimum index density and unit weight of soils and calculation of relative density. ASTM International, West Conshohocken, PA, USA.

ASTM (2007) D6528: Standard test method for consolidated undrained direct simple shear testing of cohesive soils. ASTM International, West Conshohoken, PA, USA. 
Been K and Jefferies MG (1985) A state parameter for sands. Géotechnique 35(2): 99-112.

Been K and Jefferies MG (1986) Reply to Discussion on 'A state parameter for sands'. Géotechnique 36(1): 123-132.

Been K, Jefferies MG and Hachey J (1991) The critical state of sands. Géotechnique 41(3): 365-381.

Been K, Jefferies MG and Hachey J (1992) Reply to discussion. Géotechnique 42(4): 655-663.

Been K, Jefferies MG, Hachey JE and Rothenburg L (1993) Class A prediction for model 2. In Proceedings of the International Conference on the Verification of Numerical Procedures for the Analysis of Soil Liquefaction Problems (VELACS) (Arulanandan K and Scott RF (eds)). A. A. Balkema, Rotterdam, the Netherlands.

Bjerrum L and Landva A (1966) Direct simple shear tests on a Norwegian quick clay. Géotechnique 16(1): 1-20.

Chillarige AV, Robertson PK, Morgenstern NR and Christian HA (1997) Evaluation of the in situ state of Fraser River sand. Canadian Geotechnical Journal 34(4): 510-519.

Cornforth DH (1961) Plane Strain Failure Characteristics of a Saturated Sand. PhD thesis, University of London, London, UK.

Cornforth DH (1964) Some experiments on the effect of strain condition on the strength of sand. Géotechnique 14(2): 143-167.

De Josselin de Jong G and Verruijt A (1969) Étude photoélastique d'un empilement de disques. Cahiers du Groupe Francais de Rhéologie 2(1): 73-86 (in French).

Drucker DC, Gibson RE and Henkel DJ (1957) Soil mechanics and work hardening theories of plasticity. Transactions American Society of Civil Engineers 122(1): 338-346.

Dyvik R, Berre T, Lacasse S and Raddim B (1987) Comparison of truly undrained and constant volume direct simple shear tests. Géotechnique 33(1): 3-10.

Ghafghazi M and Shuttle DA (2010) Interpretation of the in situ density from seismic CPT in Fraser River sand. Proceedings of the 2nd International Symposium on Cone Penetration Testing, Huntington Beach, CA, USA.

Gutierrez M and Ishihara K (2000) Non-coaxiality and energy dissipation in granular materials. Soils and Foundations 40(2): 49-59.

Gutierrez M, Ishihara K and Towhata I (1993) Model for the deformation of sand during rotation of principal stress directions. Soils and Foundations 33(2): 105-117.

Ishihara K and Towhata I (1983) Sand response to cyclic rotation of principal stress directions as induced by wave loads. Soils and Foundations 23(4): 11-26.

Jefferies MG (1993) NorSand: a simple critical state model for sand. Géotechnique 43(1): 91-103.

Jefferies MG (1997) Plastic work and isotropic softening in unloading. Géotechnique 47(5): 1037-1042.

Jefferies MG and Been K (2000) Implications for critical state theory from isotropic compression of sand. Géotechnique 50(4): 419-429.

Jefferies MG and Been K (2006) Soil Liquefaction, a Critical State Approach. Taylor \& Francis, Abingdon, UK.
Jefferies MG and Been K (2015) Soil Liquefaction, a Critical State Approach, 2nd edn. CRC Press, Abingdon, UK.

Jefferies MG and Shuttle DA (2002) Dilatancy in general Cambridge-type models. Géotechnique 52(9): 625-637.

Jefferies MG and Shuttle DA (2005) NorSand: features, calibration and use. In Soil Constitutive Models: Evaluation, Selection, and Calibration (Yamamuro JA and Kaliakin VN (eds)). ASCE, Reston, VA, USA, Geotechnical Special Publication no. 128, pp. 204-236.

Jefferies MG and Shuttle DA (2011) On the operating critical friction ratio in general stress states. Géotechnique 61(8): 709-713.

Kjellman W (1951) Testing the shear strength of clay in Sweden. Géotechnique 2(3): 225-232.

Konrad JM (1997) In situ sand state from CPT: evaluation of a unified approach at two CANLEX sites. Canadian Geotechnical Journal 34(1): 120-130.

Kuerbis RH and Vaid YP (1988) Sand sample preparation - the slurry deposition method. Soils and Foundations 28(4): 107-118.

Li X and Dafalias Y (2012) Anisotropic critical state theory: role of fabric. Journal of Engineering Mechanics 138(3): 263-275.

Nova R (1982) A constitutive model under monotonic and cyclic loading. In Soil Mechanics - Transient and Cyclic Loads (Pande GN and Zienkiewicz OC (eds)). Wiley, Chichester, UK, pp. 343-373.

Pestana JM and Whittle AJ (1995) Compression model for cohesionless soils. Géotechnique 45(4): 611-631.

Potts DM, Dounias GT and Vaughan PR (1987) Finite element analysis of the direct shear box test. Géotechnique 37(1): 11-23.

Rothenburg L and Bathurst RJ (1992) Micromechanical features of granular assemblies with planar elliptical particles. Géotechnique 42(1): 79-95.

Schofield AN and Wroth CP (1968) Critical State Soil Mechanics. McGraw-Hill, New York, NY, USA.

Shozen T (1991) Deformation under the Constant Stress State and its Effect on Stress-Strain Behaviour of Fraser River Sand. MASc thesis, Department of Civil Engineering, University of British Columbia, Vancouver, Canada.

Sriskandakumar S (2004) Cyclic Loading Response of Fraser River Sand for Validation of Numerical Models Simulating Centrifuge Tests. MASc thesis, Department of Civil Engineering, University of British Columbia, Vancouver, B.C., Canada.

Sukumaran B and Ashmawy AK (2002) Quantitative characterisation of the geometry of discrete particles. Géotechnique 51(7): 619-627.

Vaid YP and Negussey D (1982) A Critical Assessment of Membrane Penetration in the Triaxial Test. Soil Mechanics series no. 61. University of British Columbia, Vancouver, B.C., Canada.

Wanatowski D and Chu J (2007) Static liquefaction of sand in plane strain. Canadian Geotechnical Journal 44(3): 299-313.

Wanatowski D, Shuttle DA and Jefferies G (2013) Validation of computational liquefaction in plane strain. Proceedings of the 18th International Conference on Soil Mechanics and Geotechnical Engineering, Paris, France. 
Wong RKS and Arthur JRF (1986) Sand sheared by stresses with cyclic variation in direction. Géotechnique 36(2): 215-226.

Wride CE and Robertson PK (1997) The Canadian Liquefaction Experiment, Phase II Data Review Report, Massey and Kidd Sites, Fraser River Delta. BiTech Publishers, Richmond, B.C., Canada. Yoshimine M, Ishihara K and Vargas W (1998) Effects of principal stress direction and intermediate principal stress on undrained shear behaviour of sand. Soils and Foundations 38(3): 179-188.

Youd TL, Idriss IM, Andrus RD et al. (2001) Liquefaction resistance of soils: summary report from the 1996 NCEER and 1998 NCEER/NSF Workshops on evaluation of liquefaction resistance of soils. Journal of Geotechnical and Geoenvironmental Engineering ASCE 127(10): 817-833.

\section{WHAT DO YOU THINK?}

To discuss this paper, please submit up to 500 words to the editor at journals@ice.org.uk. Your contribution will be forwarded to the author(s) for a reply and, if considered appropriate by the editorial panel, will be published as a discussion in a future issue of the journal. 